\title{
The rate dependent response of a bistable chain at finite temperature
}

\author{
Itamar Benichou ${ }^{1}$, Yaojun Zhang ${ }^{2}$, Olga K. Dudko ${ }^{3}$, Sefi Givli ${ }^{1 *}$ \\ ${ }^{1}$ Faculty of Mechanical Engineering, Technion - Israel Institute of Technology, Haifa 32000, Israel \\ ${ }^{2}$ Princeton Center for Theoretical Science, Princeton University, Princeton, New Jersey 08544, USA \\ ${ }^{3}$ Department of Physics, University of California at San Diego, La Jolla, CA, USA \\ *Corresponding author, email: givli@technion.ac.il
}

\begin{abstract}
We study the rate dependent response of a bistable chain subjected to thermal fluctuations. The study is motivated by the fact that the behavior of this model system is prototypical to a wide range of nonlinear processes in materials physics, biology and chemistry. To account for the stochastic nature of the system response, we formulate a set of governing equations for the evolution of the probability density of meta-stable configurations. Based on this approach, we calculate the behavior for a wide range of parametric values, such as rate, temperature, overall stiffness, and number of elements in the chain. Our results suggest that fundamental characteristics of the response, such as average transition stress and hysteresis, can be captured by a simple law which folds the influence of all these factors into a single non-dimensional quantity. We also show that the applicability of analytical results previously obtained for single-well systems can be extended to systems having multiple wells by proper definition of rate and of the transition stress.
\end{abstract}

Keywords: Thermal fluctuations, energy barriers, bistable chain, hysteresis, transition stress. 


\section{Introduction}

The saw-tooth pattern appearing in a (generalized) force-displacement response is the fingerprint of transition processes involving discrete switching events between metastable states. This phenomenon, sometimes termed "discrete phase transformation” (Benichou and Givli, 2013; Manca et al., 2014), is prevalent in smallscale systems and is also a mechanism responsible for a large number of non-linear processes at the macro scale. Examples from materials physics include the behavior of ferromagnetic alloys (Benichou et al., 2013), nano-indentation (Dan Mordehai, 2011; Maaß et al., 2012; Wang et al., 2012), nanoslabs of nickel under compression (Pattamatta et al., 2014), uniaxial compression of nanopillars (Jennings et al., 2011; Kunz et al., 2011) and plasticity (Müller and Villaggio, 1977; Puglisi and Truskinovsky, 2002a; Q.P. Sun, 2008; Seelecke et al., 2005). Another important class of materials exhibiting a saw-tooth pattern in force-extension experiments are macromolecular materials, such as DNA (Bosaeus et al., 2012; Gross et al., 2011; King et al., 2013; Ritwik Raj, 2011), spider silk, biopolymers, polysoaps, and artificial elastomers (Benichou and Givli, 2011; Chyan et al., 2004b; Kellermayer et al., 1997; Labeit et al., 2003; Oberhauser, 2001; Oberhauser et al., 2001; Oberhauser et al., 1998; Rief et al., 1997; Rief et al., 1998; Schlierf et al., 2004; Schwaiger et al., 2002; Tskhovrebova et al., 1997; Williams et al., 2003). In proteins for example, this is constituted by elastic (entropic) macromolecules reinforced by a finite number of stiff domains, usually in the form of fillers or $\beta$-sheets. These domains undergo hardsoft transitions by unfolding into soft (entropic) domains. These discrete switching events give rise to the saw-tooth pattern observed in single molecule force-extension experiments (Benichou and Givli, 2011; Chyan et al., 2004a; Gross et al., 2011; Oberhauser et al., 2002; Rief et al., 1997). Finally, many-particle reversible storage systems, such as lithium-ion batteries (Dreyer et al., 2011; Dreyer et al., 2010), also exhibit a similar behavior.

A large number of works, some date back to the 1970's (Müller and Villaggio, 1977), have demonstrated that the non-trivial behavior of an elastic chain composed of bistable elements is prototypical for such processes. Most of these works focused on purely static analysis, clarifying issues such as stability and the role of the spinodal region (Puglisi and Truskinovsky, 2000), investigating the effects of interactions beyond the nearest neighbors (Rogers and Truskinovsky, 1997), the behavior in more 
than one dimension (Kinderlehrer and Ma, 1994; Müller and Villaggio, 1977), and the influence of various types of non-convex springs ranging from Lennard-Jones type potentials (Braides et al., 1999; Truskinovsky, 1996) to tri-parabolic and bi-parabolic approximations of the spring energy (Fedelich and Zanzotto, 1992; Puglisi and Truskinovsky, 2002b).

The dynamic behavior of bistable chains poses a real theoretical challenge, and indeed fewer studies investigated the dynamics of bistable chains. These studies can be roughly divided into two main categories. The first involves modeling inertial dynamics directly, e.g. (Balk et al., 2001; Cherkaev et al., 2005; Cohen and Givli, 2014; Efendiev and Truskinovsky, 2010; Slepyan and Troyankina, 1984; Vainchtein, 2010; Zhao and Purohit, 2014). A second approach is to obtain the dynamic response by assuming simplified evolution strategies or kinetic relations, e.g. (Givli, 2010; Givli and Bhattacharya, 2009; Puglisi and Truskinovsky, 2002b; Ritwik Raj, 2011).

In nanoscale structures, thermal fluctuations often govern the dynamic response by enabling overcoming energy barriers separating between metastable configurations. Roughly, the response is divided into three regions of rates: at very low rates, the system operates at quasi-equilibrium; at moderate rates thermal noise dominates the behavior, resulting in a stochastic response; and at sufficiently high rates, the system has no time to explore its energy landscape, so stochastic fluctuations become irrelevant and the response is deterministic (Rico et al., 2013). This means that in the passage from low to intermediate and then to high rates the system experiences transitions from deterministic to stochastic and back to deterministic response. In addition, fundamental differences distinguish between these three regions. For example, in the first region (low rates), the system exhibits no hysteresis and transition stresses are lowest. Hysteresis appears in the other two regions, but at intermediate rates it is a consequence of transitions from high-energy to low-energy configurations, while at very high rates it is dominated by a standard damping mechanism.

The rates defining each region may vary significantly, depending on specific features of the energy landscape. Hence, establishing a universal scale that uniquely determines whether a rate is high, low or intermediate is of key importance. Such a scale can also facilitate quantitative predictions of fundamental features, such as hysteresis and transition stress. Addressing this challenge is the main goal of this paper. To this end, we consider the model system of an elastic chain composed of 
bistable elements connected in series, and study its rate-dependent response at finite temperature. Having in mind nano scale structures and macromolecular materials, the height of energy barriers separating between meta-stable configurations is typically of the order of ten to a few hundreds $k_{B} T$, making the system susceptible to thermal noise. In addition, the dynamics of these systems is overdamped, meaning that excess energy associated with switching events completely dissipates into heat.

Experimental investigation of micro-scale structures or macromolecular materials typically involve mechanically induced switching of material domains by techniques such as AFM, optical tweezers, or magnetic tweezers (Brown et al., 2007; Linke and Grutzner, 2008; Oberhauser et al., 2001; Rief et al., 1997; Shulha et al., 2006; Wang et al., 2012). In these experiments, the mechanical load is usually set by moving the experimental stage at some predefined rate. This length constraint introduces mechanical coupling between domains, and dictates a response that is fundamentally different from force-control (dead load/soft device) experiments. In length-control conditions (hard device), energy barriers separating between configurations depend on the overall strain, but also on the overall compliance and on the configuration (number of domains that already switched). On the other hand, in force-control experiments, the energy barriers separating between meta-stable configurations depend on the level of external force but are indifferent to the overall compliance and configuration (Puglisi and Truskinovsky, 2000). Since energy barriers dominate the rate dependent and temperature dependent dissipative behavior of systems susceptible to thermal noise, one must account for the actual loading conditions when analyzing such experiments. The above discussion regarding the difference between forcecontrol and length-control conditions resembles a similar discussion on stretching polymer chains (without domains). It has been shown that different boundary conditions (Helmholtz or Gibbs ensembles) imposed for stretching the polymer lead to different force-extension curves (Keller et al., 2003; Manca et al., 2012b; Sinha and Samuel, 2005). These differences are emphasized when the contour length of the molecule is of the same order of its persistence length, and become indistinguishable at the thermodynamic limit (Winkler, 2010).

Theoretical studies concerning the thermo-mechanical behavior of multi-stable chains susceptible to thermal noise have taken various approaches, e.g. (Achenbach and Muller, 1982; Brown et al., 2009; Evans and Ritchie, 1997; Evans and Ritchie, 
1999; Huo and Müller, 1993; King et al., 2010; Manca et al., 2012a, 2013; Mirny and Shakhnovich, 2001; Muller and Seelecke, 2001; Purohit et al., 2011; Singh et al., 2011; Staple et al., 2009; Su and Purohit, 2009). These works can be roughly divided into three categories: formulation of mathematical models based on two-state theory, a continuum approach based on a Fokker-Planck formulation (Herrmann et al., 2012), and intensive computer based simulations, such as Langevin dynamics (Bonilla et al., 2015; Plata et al., 2015), molecular dynamics (MD) and all-atom Monte-Carlo approaches. MD theories have been restricted by the computational effort required to describe the behavior of large macromolecules at the typical (experimental) loading time scale. On the other hand, two-state theories often take a phenomenological approach or adopt simplifying assumptions that limit the applicability of the model to a specific range of loading rates and to, often unintentionally, force-control conditions. We note that analytical approximations have been found for the simple case of systems with a single energy well and irreversible energy barrier which decreases monotonously in time to zero, e.g. (Dudko, 2015; Dudko et al., 2006; Evans and Ritchie, 1999; Garg, 1995). In particular, closed form expressions were derived for the average and variance of the transition stress (rupture force in the context of molecular bonds) by means of asymptotic approximations. We show that by identifying the right reference energy and time scales combined with a suitable definition of the transition stress, the applicability of analytical insights obtained for single-well systems can be extended for more general cases.

In this paper, we adopt the following strategy for investigating the rate-dependent dissipative behavior of bistable chains subjected to thermal fluctuations. The stochastic nature of the system response is translated into a set of governing equations for the evolution of the probability density of meta-stable configurations. Based on this approach, we calculate the behavior for a wide range of parametric values, such as rate, temperature, overall stiffness, and number of elements. We show that the fundamental characteristics of the response can be captured by a simple universal law which folds the influence of all these factors into a single non-dimensional quantity. Importantly, our analysis accounts for two prominent features which are usually overlooked. First, we account for the simultaneous existence of several meta-stable configurations at a prescribed overall strain. Second, we rigorously take into consideration the fact that hard device (displacement-control/Helmholtz-ensemble) conditions are fundamentally different from soft device (force-control, Gibbs- 
ensemble). Some of the results of this paper have been announced in a pre-publication (Benichou and Givli, 2015). Here, we provide additional details, and include new results and further insights. In particular, while the focus of (Benichou and Givli, 2015) was merely on effects of rate and temperature on the average hysteresis, we extend here the results to the study of average transition stress and its dispersion. Importantly, we show that the relevant measurable should be the transition time rather than hysteresis. Once transition time is obtained, statistical properties of the transition stress can be directly computed by means of straight-forward transformation that involves the externally-controlled ramp rate. Further, we provide analytical expressions and justifications to the main numerical conclusions. Importantly, we show that by adopting the correct experiment time scale, the results and insights can be extended to all types of bistable elements, not necessarily tri-linear ones, and to any type of loading conditions, either hard device (displacement-control/Helmholtzensemble) or soft device (force-control, Gibbs-ensemble).

\section{Theoretical considerations}

Consider the overdamped dynamics of a 1D chain subjected to thermal fluctuations. The chain is composed of $N$ identical bistable elements connected in series. It is fixed at one end and extended at a prescribed rate $v=d x / d t$ at the other end (hard device). The overall strain of the chain is $\varepsilon=x / L$, where $L=N a$ is the unloaded length, and $a$ is the undeformed length of each element. Each bistable element is characterized by a non-convex energy landscape having two disjoint convex intervals (wells) separated by a spinodal region with concave energy. We term the convex intervals "Phase-I" and "Phase-II" (Muller and Xu, 1991; Puglisi and Truskinovsky, 2000). The resulting stress-strain relation is not monotonous, as illustrated in Fig. 1. Consequently, the system allows for a variety of nontrivial equilibrium configurations characterized by an inhomogeneous strain. In other words, at a given overall strain, several equilibrium configurations associated with local energy minima may exist. These configurations differ by the number of elements in Phase-II, which we denote by $n$.

At finite temperature, $T>0$, the dynamic behavior of the overdamped system is governed by thermal fluctuations. If the thermal energy $k_{B} T$ is much smaller compared to the energy barriers separating between configurations (local energy 
minima), the response involves long "waiting” periods around the local minima followed by sudden jumps from one meta-stable configuration to another. In this case, the escape rate depends on the saddle point (lowest barrier) between energy minima, and can be approximated by Kramers theory (Hänggi et al., 1990; Suess and Fidler, 2006). Although the (Helmholtz) free energy landscape, $F$, may be complex, we take the simplified view that each configuration is positioned along a specific reaction path in which the configurations are arranged in a sequential order. This path defines a 1D energy landscape that includes multiple minima separated by barriers associated with the lowest saddle points mentioned above, as illustrated in Fig. 2. Note that the topography of this landscape depends on the overall strain, thus time dependent. We further assume that the system is adjusted instantaneously to this time dependent energy landscape, i.e. that the time scale of intra-well relaxation is faster than other time scales of the system (Friddle et al., 2012).

In order to account for the stochastic nature of the response, we denote by $P_{n}$ the probability of finding the system in configuration $n$. The evolution in time of the probability $P_{n}$ is governed by the following set of $N+1$ ordinary differential equations

$$
\frac{d P_{n}}{d t}=I_{n-1}^{+}-I_{n}^{-}-I_{n}^{+}+I_{n+1}^{-} \quad(n=0,1,2, \ldots, N)
$$

Here, $I_{n}^{ \pm}$describe the probability fluxes from configuration $n$ to configurations $n \pm 1$, respectively, as illustrated in Fig. 2. Once $P_{n}$ is obtained, the (ensemble) average stress-strain relation is found by

$$
\langle\sigma(\varepsilon)\rangle=\sum_{n=0}^{N} \sigma_{n}(\varepsilon) P_{n}(\varepsilon)
$$

where $\sigma_{n}(\varepsilon)$ is the stress associated with configuration $n$ at overall strain $\varepsilon=\varepsilon(t)$. Similarly, the variance of the stress, $\left\langle\sigma^{2}\right\rangle-\langle\sigma\rangle^{2}$, and higher statistical moments can be calculated using:

$$
\left\langle\sigma^{m}\right\rangle=\sum_{n=0}^{N}\left[\left(\sigma_{n}\right)^{m} P_{n}\right] .
$$


The probability flux may be written as $I_{n}^{ \pm}=r_{n}^{ \pm} P_{n}$, where $r_{n}^{ \pm}$are the escape rates from configuration $n$ to configurations $n \pm 1$. Thus, Eq. (2.1) becomes

$$
\frac{d P_{n}}{d t}=r_{n-1}^{+} P_{n-1}-\left(r_{n}^{-}+r_{n}^{+}\right) P_{n}+r_{n+1}^{-} P_{n+1}
$$

According to Kramers theory $r_{n}^{ \pm}(t)=r_{0} \exp \left(-\Delta F_{n}^{ \pm}(t) / k_{B} T\right)$ (Risken, 1984), i.e.

$$
\frac{d P_{n}}{d t}=r_{0}\left[e^{-\Delta F_{n-1}^{+} / k_{b} T} P_{n-1}-\left(e^{-\Delta F_{n}^{-} / k_{b} T}+e^{-\Delta F_{n}^{+} / k_{b} T}\right) P_{n}+e^{-\Delta F_{n+1}^{-} / k_{b} T} P_{n+1}\right]
$$

Above,

$$
r_{0}=\frac{\sqrt{k\left|k^{*}\right|}}{2 \pi \eta L}
$$

is the attempt frequency (Hänggi et al., 1990), where $k$ and $k^{*}$ are the stiffness ( $\partial \sigma / \partial \varepsilon$ ) at the minimum and the saddle points, respectively, and $\eta$ is the damping coefficient. $\Delta F_{n}^{+}=\left.\left(F_{n}^{*}-F_{n}\right)\right|_{\varepsilon(t)}$ and $\Delta F_{n}^{-}=\left.\left(F_{n-1}^{*}-F_{n}\right)\right|_{\varepsilon(t)}$ are the minimal free energy barriers separating between configuration $n$ to configurations $n+1$ and $n-1$. Throughout, ()$_{n}$ and ()$_{n}^{*}$ denote quantities associated with configuration $n$ (substantial configuration) and with the saddle point separating configurations $n$ and $n+1$ (transitional configuration), respectively. For instance, $F_{n}$ is the free energy of configuration $n$ at overall strain $\varepsilon$, and $F_{n}^{*}$ is the free energy associated with the saddle point separating configurations $n$ and $n+1$ at the same overall strain. This saddle point involves exactly one element in the spinodal region (Benichou and Givli, 2013; Puglisi and Truskinovsky, 2000).

The free energy is the sum of the internal energy and the entropy contribution, $F=U-k_{B} T \ln \Omega$, where $\Omega$ is the number of arrangements (microstates) for a given configuration (macrostate). In particular (Muller and Seelecke, 2001)

$$
\Omega_{n}=\frac{N !}{n !(N-n) !}, \quad \Omega_{n}^{*}=\frac{N !}{n !(N-n-1) !},
$$

and therefore

$$
\Delta F_{n}^{+}=B_{n}^{+}-k_{B} T \ln (N-n), \Delta F_{n}^{-}=B_{n}^{-}-k_{B} T \ln (n) .
$$


Above,

$$
B_{n}^{+}=\left.\left(U_{n}^{*}-U_{n}\right)\right|_{\varepsilon(t)} \text { and } B_{n}^{-}=\left.\left(U_{n-1}^{*}-U_{n}\right)\right|_{\varepsilon(t)}
$$

are the internal energy barriers, or the energy barriers at zero temperature.

For a constant extension rate, $v$, three time scales govern the chain dynamics: (i) the average time between escape attempts, $t_{0}=1 / r_{0}$, (ii) the average time between successful escapes over a (free energy) barrier of a typical height, $t_{M}$, (iii) the time the chain spends in a meta-stable configuration before switching to the next, $t_{c}$, which sets the experiment time scale. We define the non-dimensional time as

$$
\hat{t}=\frac{t}{t_{c}}
$$

Also, the energy scale of a single bistable element, $U_{c}$, is used to define nondimensional energies

$$
\hat{F}=F / U_{c} .
$$

Accordingly, the governing equations (2.5) take the following non-dimensional form:

$$
\frac{d P_{n}}{d \hat{t}}=\frac{1}{\omega}\left[(N-n+1) e^{-\hat{\beta} \hat{B}_{n-1}^{+}} P_{n-1}-\left(n^{-\hat{\beta} \hat{B}^{-}}+(N-n) e^{-\hat{\beta} \hat{B}_{n}^{+}}\right) P_{n}+(n+1) e^{-\hat{\beta} \hat{B}_{n+1}^{-}} P_{n+1}\right] .
$$

The entropic contribution in (2.8) appears as a pre-exponential factor that reflects the number of possibilities to change from the relevant configuration. For example, at configuration $n, N-n$ elements are in Phase-I and $n$ elements are in Phase-II. Thus, changing to configuration $n-1$ is possible by switching any of the $n$ elements that are in Phase-II to Phase-I, while changing to configuration $n+1$ can take place by switching to Phase-II any of the $N-n$ elements that are in Phase-I. In (2.12), we have introduced the following non-dimensional quantities

$$
\omega \equiv t_{0} / t_{c}, \hat{\beta} \equiv U_{c} / k_{B} T, \hat{B}_{n} \equiv B_{n} / U_{c} .
$$

$\omega$ is proportional to the velocity $v$, thus serves as a non-dimensional rate. $\omega^{-1}$ roughly describes the number of escape attempts during the experiment time scale $t_{c}$. For most practical cases $\omega<1 . \hat{\beta}$, which describes the size of the single element, 
acts as a scaling parameter on the non-dimensional barriers, $\hat{B}_{n}$, which are timedependent through overall strain. Together, the term $\hat{\beta} \hat{B}_{n}$ describes the energy barrier in units of $k_{B} T$.

Although Eq. (2.12) cannot be solved analytically, several insights can be obtained from its structure. First, note that at extremely low rates, i.e. $\omega \rightarrow 0$, the right-handside of (2.5), or alternatively (2.12), must vanish. It is easy to verify that in this situation the solution for $P_{n}$ reduces to Boltzmann distribution. Also, the dynamic behavior of the system is governed by the three non-dimensional parameters, $\omega, N$, $\hat{\beta}$, which define rate and size. The details of the specific system under consideration are reflected solely in the non-dimensional barriers $\hat{B}_{n}$. For example, the particular shape (tri-linear, cubic, etc.) of the stress-strain relation, Fig. 1, only affects $\hat{B}_{n}$. A different shape will alter some details of the solution, but is not expected to fundamentally change the essence of the predicted behavior. Therefore, for specificity and without loss of generality, we consider next the case of a chain composed of trilinear elements.

\section{A chain composed of tri-linear elements}

A tri-linear element is a bistable element that has an energy per unit length, $u(\varepsilon)$, characterized by two quadratic wells separated by a quadratic spinodal region. Consequently, the corresponding stress-strain relation, $\sigma(\varepsilon)=d u / d \varepsilon$, takes a trilinear form. This relation is governed by five parameters: $k^{I}, k^{I I}, \sigma_{\max }, \sigma_{\min }, A$, as illustrated in Fig. 3. $k^{I}, k^{I I}$ are the stiffness of the bistable element in Phase-I and Phase-II, respectively, $\sigma_{\max }$ is the maximal stress that can be attained in Phase-I, $\sigma_{\text {min }}$ is the minimal stress that can be attained in Phase-II, and $A$ is the transition strain at $\sigma_{\min }$. For convenience, we define $k^{I}=k, k^{I I}=\lambda k, \Delta \sigma=\sigma_{\max }-\sigma_{\min }$. The parameter $\lambda$ determines whether the system is hardening $(\lambda>1)$, neutral $(\lambda=1)$ or softening $(\lambda<1)$. 
The energy-strain relation of configuration $n, u_{n}(\varepsilon)$, and the corresponding stress-strain relation, $\sigma_{n}(\varepsilon)$, can be described by the following set of segments (Benichou and Givli, 2013):

$$
\begin{aligned}
& u_{n}\left(\sigma_{n}(\varepsilon)\right)=\frac{\left(\sigma_{n}(\varepsilon)\right)^{2}}{2 K_{n}}-\frac{1}{2}\left(\frac{1}{k}-\frac{1}{K_{n}}\right) \sigma_{\min }^{2}+\frac{1}{2}\left(\sigma_{\min }+\sigma_{\max }\right) A \frac{n}{N} \\
& \sigma_{n}(\varepsilon)=K_{n}\left[\varepsilon-\left(\frac{1}{k}-\frac{1}{K_{n}}\right) \sigma_{\min }-A \frac{n}{N}\right]
\end{aligned}
$$

where

$$
K_{n}=k\left[1+\left(\frac{1}{\lambda}-1\right) \frac{n}{N}\right]^{-1} .
$$

Each of these segments is defined on an interval of the overall strain $\varepsilon$ such that $\sigma_{\min } \leq \sigma_{n}(\varepsilon) \leq \sigma_{\max }$. Similarly, expressions for the "transitional" configurations, $u_{n}^{*}(\varepsilon)$ and $\sigma_{n}^{*}(\varepsilon)$, which are associated with the saddle-points separating between local energy minima take the form:

$$
\begin{aligned}
& u_{n}^{*}\left(\sigma_{n}(\varepsilon)\right)=\frac{\left(\sigma_{n}^{*}(\bar{\varepsilon})\right)^{2}}{2 K_{n}^{*}}-\frac{1}{2}\left(\frac{1}{k}-\frac{1}{K_{n}}\right) \sigma_{\min }^{2}+\frac{1}{2}\left(\sigma_{\min }+\sigma_{\max }\right) \frac{A n}{N}+\frac{1}{2} \frac{\sigma_{\max }^{2}}{\Delta \sigma} \frac{A}{N} \\
& \sigma_{n}^{*}(\varepsilon)=K_{n}^{*}\left[\varepsilon-\left(\frac{1}{k}-\frac{1}{K_{n}^{*}}\right) \sigma_{\text {min }}-A \frac{n+1}{N}\right]
\end{aligned}
$$

where

$$
K_{n}^{*}=k\left[1+\left(\frac{1}{\lambda}-1\right) \frac{n}{N}-\frac{k A}{N \Delta \sigma}\right]^{-1} .
$$

The energy scale of the tri-linear element is set by $U_{c}=a \cdot A \Delta \sigma$. In addition, it is convenient to define the normalized stress $\hat{\sigma}=\sigma / \Delta \sigma$, strain $\hat{\varepsilon}=\varepsilon / A$, and stiffness $\hat{K}_{n}=K_{n} / k$. Thus, $\hat{K}_{n}$ varies from one for $n=0$ to $\lambda$ for $n=N$, and $\phi \hat{K}_{n}=d \hat{\sigma}_{n} / d \hat{\varepsilon}$ where $\phi \equiv k A / \Delta \sigma$. Using these definitions we write 


$$
\begin{aligned}
& \hat{U}_{n}=\frac{U_{n}}{U_{c}}=\frac{a N u_{n}}{U_{c}}=\frac{1}{2}\left[\frac{\hat{\sigma}_{n}^{2}}{\phi \hat{K}_{n}}+\left(1-\frac{1}{\hat{K}_{n}}\right) \frac{\hat{\sigma}_{\text {min }}^{2}}{\phi}+\left(1+2 \hat{\sigma}_{\text {min }}\right) \frac{n}{N}\right] N \\
& \hat{U}_{n}^{*}=\frac{U_{n}^{*}}{U_{c}}=\frac{a N u_{n}^{*}}{U_{c}}=\frac{1}{2}\left[\frac{\hat{\sigma}_{n}^{* 2}}{\phi \hat{K}_{n}^{*}}+\left(1-\frac{1}{\hat{K}_{n}}\right) \frac{\hat{\sigma}_{\text {min }}^{2}}{\phi}+\left(1+2 \hat{\sigma}_{\text {min }}\right) \frac{n}{N}+\left(1+\hat{\sigma}_{\text {min }}\right)^{2} \frac{1}{N}\right] N
\end{aligned}
$$

where

$$
\begin{aligned}
& \hat{\sigma}_{n}(\hat{\varepsilon})=\phi \hat{K}_{n}\left[\hat{\varepsilon}-\left(1-\frac{1}{\hat{K}_{n}}\right) \frac{\hat{\sigma}_{\min }}{\phi}-\frac{n}{N}\right] \\
& \hat{\sigma}_{n}^{*}(\hat{\varepsilon})=\phi \hat{K}_{n}^{*}\left[\hat{\varepsilon}-\left(1-\frac{1}{\hat{K}_{n}^{*}}\right) \frac{\hat{\sigma}_{\min }}{\phi}-\frac{n+1}{N}\right]
\end{aligned}
$$

Finally, by plugging (3.5) into (2.9) we conclude with the following expressions for the non-dimensional barriers:

$$
\begin{aligned}
& \hat{B}_{n}^{+}=\frac{1}{2}\left[\frac{\hat{\sigma}_{n}^{* 2}}{\phi \hat{K}_{n}^{*}}-\frac{\hat{\sigma}_{n}^{2}}{\phi \hat{K}_{n}}+\left(1+\hat{\sigma}_{\min }\right)^{2} \frac{1}{N}\right] N \\
& \hat{B}_{n}^{-}=\frac{1}{2}\left[\frac{\hat{\sigma}_{n-1}^{* 2}}{\phi \hat{K}_{n-1}^{*}}-\frac{\hat{\sigma}_{n}^{2}}{\phi \hat{K}_{n}}+\frac{\hat{\sigma}_{\min }^{2}}{N}\left(\frac{1-\hat{K}_{n}}{\phi \hat{K}_{n}} \frac{N}{n}+1\right)\right] N
\end{aligned}
$$

These energy barriers change in time since $\hat{\sigma}_{n}$ and $\hat{\sigma}_{n}^{*}$ depend on the overall strain $\hat{\varepsilon}=\hat{\varepsilon}(t)$. The barriers $\hat{B}_{n}^{+}$monotonously decrease with strain, while $\hat{B}_{n}^{-}$ monotonously increase. The above expressions significantly simplify for neutral systems $(\lambda=1)$ since in that case $\hat{K}_{n}=1$ and $\hat{K}_{n}^{*}=(1-\phi / N)^{-1}$ for all $n$.

Fig. 4 shows the stress-strain and energy-strain relations for a chain composed of four elements. Each of the segments in Fig. $4 \mathrm{~b}$ is quadratic with respect to $\hat{\varepsilon}$, where $\hat{U}_{n}$ and $\hat{U}_{n}^{*}$ are distinguished by the solid and dashed lines, respectively. Note that for a prescribed overall strain, several configurations are possible. For example, at $\hat{\varepsilon}=\hat{\varepsilon}_{0}$, there are three possible meta-stable configurations, $n=0,1,2$, which are marked by the filled circle, square and triangle, respectivelly. At this specific strain, the lowest energy is associated with configuration $n=1$. In the presence of thermal fluctuations the system will have tendency towards this configuration. Yet, the chain may be found in any of the other two configurations as well. The energy barriers separating between configurations are illustrated in Fig. 4c as function of overall 
strain. A few comments are in order. Since $\hat{U}_{n}$ and $\hat{U}_{n}^{*}$ are both quadratic functions of $\hat{\varepsilon}$, (2.9) suggests that the energy barriers, $\hat{B}_{n}^{+}$and $\hat{B}_{n}^{-}$, are quadratic functions of $\hat{\varepsilon}$ as well. The consequences of this feature will be further discussed in Section 5 . Note also that the barriers (3.7) depend on the number of elements in the chain, $N$, and on the configuration $n$, in contrast to soft device conditions (Puglisi and Truskinovsky, 2002b). Importantly, the commonly used Bell-type approximation (Bell, 1978; Zhurkov, 1965), postulating that the barrier is lowered in proportion to the external force, is valid only for soft device conditions with highly curved barriers, and is not suitable for hard device experiments.

The sign of $\hat{K}_{n}^{*}$ (or of $1-\phi / N$ in the case of $\lambda=1$ ) holds important information regarding the system behavior. Positive values indicate that transitional configurations, i.e configurations involving exactly one element in the spinodal region, are unstable (Puglisi and Truskinovsky, 2000). On the other hand, negative values indicate that the transitional configurations are stable. In this case, exactly one configuration exists for any overall strain, thus energy barriers do not exist (which is manifested by negative values of $\hat{B}_{n}$ in (3.7)), $P_{n}$ takes values of either zero or one, and the response is deterministic. Therefore, we focus our attention to case of $\hat{K}_{n}^{*}<0$. Finally, in accordance with Section 2, $t_{c}$ describes the typical time the system remains in a configuration, i.e.

$$
t_{c}=A a / v \text {. }
$$

\section{Results}

In this section, we present numerical solutions of equation (2.12) for tri-linear chains. In particular, we are interested in understanding the effects of the various parameters on the rate dependent and temperature dependent behavior of the system.

\subsection{Average transition stress}

Fig. 5 shows a set of typical stress-strain relations. The thin lines illustrate the stressstrain segments (3.6), while the thick lines illustrate the average (most probable) behavior, (2.2). The different colors correspond to either different temperatures or rates, as explained below. The protocol of each experiment involves two stages of pull 
and release at the same constant rate. First, the chain is extended from configuration $n=0$ until all elements switch to Phase-II (loading path). The stress at which the chain switches to the next configuration is termed the loading transition stress, $\hat{\sigma}_{\max }^{t}$. Next, the length of the chain is reduced at the same rate (with opposite sign) from configuration $n=N$ back to $n=0$, i.e. unloading path. We define the stress at which switching occurs as the unloading transition stress, $\hat{\sigma}_{\min }^{t}$. In what follows, unless stated otherwise, we use the term "transition stress" in the context of loading transition stress. Higher rates or lower temperatures increase the transition stress. This is intuitively understood, since at lower temperatures the magnitude of the thermal fluctuations is smaller, thus the probability of escaping barriers is smaller. Similarly, at higher rates, there is a higher probability for reaching a higher stress level before escaping from the current configuration. If the rate is high enough (or temperature low enough), the system follows the maximum hysteresis path, illustrated in red. On the other hand, for very low rates (or high temperatures) the system follows the equilibrium path, where loading and unloading paths merge, and there is no hysteresis, as illustrated by the black line.

The following discussion and ideas related to the transition stress are not limited to a particular system. All results can be generalized to non-neutral chains $(\lambda \neq 1)$, but these involve cumbersome expressions. Thus, for simplicity and without loss of generality, we chose to focus our attention below to the neutral chain $(\lambda=1)$. As mentioned earlier, Maxwell's path is the global energy minimizer. On this path, switching from configuration $n$ to configuration $n+1$ or from configuration $n+1$ back to configuration $n$ take place at the same strain, $\hat{\varepsilon}_{M}$, as illustrated in Fig. 6. For brevity, we do not explicitly indicate the dependence of $\hat{\varepsilon}_{M}$ on $n$. The corresponding transition stresses, termed Maxwell's stresses, are $\hat{\sigma}_{M}^{+}$and $\hat{\sigma}_{M}^{-}$, respectively. It is easy to verify that the difference between these stresses $\hat{\sigma}_{M}^{+}-\hat{\sigma}_{M}^{-}=\phi / N$. As expected, this difference vanishes at the continuum limit where $N \rightarrow \infty$. During the loading phase, lower rates lead to lower transition stresses. Practically, the lowest average transition stress, corresponding to quasi-equilibrium, is $\sigma_{M}^{+}$. This important feature implies that the average transition stress may take values between $\sigma_{M}^{+}$, at low 
rates, to $\sigma_{\max }$ at high rates. Hence, the reference point from which the transition stress should be measured is $\sigma_{M}^{+}$. Accordingly, we define the normalized transition stress as

$$
\bar{\sigma}^{t}=\frac{\hat{\sigma}_{\max }^{t}-\hat{\sigma}_{M}^{+}}{\hat{\sigma}_{\max }-\hat{\sigma}_{M}^{+}},
$$

which varies from zero, at very low rates, to one at very high rates. We emphasize that the concept of measuring the transition stress relative to the Maxwell's stress is indispensable to the analysis of chains with multiple energy wells (sequential barriers). The importance and implications of this concept are further discussed in Section 5. Note that in simpler models involving a single energy well with irreversible barrier, the notion of Maxwell's stress is irrelevant and transition stress is usually measured relative to the arbitrary starting point of zero stress, e.g. (Evans and Ritchie, 1999; Garg, 1995).

Next, we quantitatively study the effects of rate, $\omega$, and the relative height of typical barriers (also inversely proportional to temperature), $\hat{\beta} \hat{B}_{M}$, on the transition stress $\bar{\sigma}^{t}$. Fig. 7a shows $\bar{\sigma}^{t}$ for a chain composed of ten elements with $\lambda=1$, $\phi=1.25, \hat{\sigma}_{\min }=-\hat{\sigma}_{\max }$ for various values of $\omega$ and $\hat{\beta} \hat{B}_{M}$. As expected, the transition stress is monotonously increasing with $\omega$ and $\hat{\beta} \hat{B}_{M}$. The extreme value of $\bar{\sigma}^{t}=0$ can only be obtained for very low rates (or high temperatures), e.g. $\omega<10^{-24}$ for $\hat{\beta} \hat{B}_{M}=50$. In other words, the rate is small enough such that the system is practically in a state of thermodynamic equilibrium at all times. The other extreme case is $\bar{\sigma}^{t}=1$, which is associated with rates of $\omega \square 1$ or higher, regardless of $\hat{\beta} \hat{B}_{M}$. Recall that $\omega^{-1}$ describes the number of escape attempts during the experiment time scale $t_{c}$. Thus, for $\omega>1$ the number of attempts is so small that overcoming barriers is practically impossible, the behavior is deterministic and the system effectively follows the maximum hysteresis path. Note that the contribution of the damping force, $\eta v$, may not be negligible for such extreme rates. Hence, relation (2.2) is valid only when the contribution of the damping force is small compared to the typical stress, i.e. the "rate condition" $\eta v \square \Delta \sigma$ holds. This condition, which can also be interpreted as assuming that the system is adjusted instantaneously to the time-dependent energy landscape, practically limits the rate to $\omega<1$, as will be explained later. Thus, we 
focus our attention to the more interesting range of $\omega<1$. It is evident that in this range $\omega$ cannot be used as a sole measure for appreciating whether the system is close to equilibrium or to the maximum hysteresis, since hysteresis is significantly varied by $\hat{\beta} \hat{B}_{M}$. Thus, instead of the ratio $t_{0} / t_{c}$, it may be more useful to introduce the ratio

$$
\tau=t_{M} / t_{c},
$$

which roughly describes the number of escapes over a typical barrier, $\hat{\beta} \hat{B}_{M}$, during the experiment time scale $t_{c}$. A natural choice of the typical energy barrier is associated with the Maxwell's path, i.e. the barrier separating two neighboring configurations at the strain $\varepsilon_{M}$, corresponding to equal energies of the two configurations. For a chain of bistable elements we write

$$
t_{M}=t_{0} e^{\hat{\beta} \hat{B}_{M}-\ln N / 2}=\frac{t_{0}}{N / 2} e^{\hat{\beta} \hat{B}_{M}}
$$

where the term $\ln (N / 2)$ accounts for the entropy at the "midway" configuration $n=N / 2$, and $\hat{B}_{M}$ is the energy barrier associated with the Maxwell path at zero temperature. In the case of $\lambda=1, \hat{B}_{M}$ simply reads

$$
\hat{B}_{M}=\frac{1}{8}\left(1-\frac{\phi}{N}\right)
$$

Otherwise, the closed form of $\hat{B}_{M}$ takes a more cumbersome expression (Benichou and Givli, 2013). Plugging (4.3) into (4.2) we have that

$$
\tau=\frac{\left(2 t_{0} / N\right) e^{\hat{\beta} \hat{B}_{M}}}{t_{0} / \omega}=\frac{\omega}{N / 2} e^{\hat{\beta} \hat{B}_{M}} .
$$

The results of Fig. 7a are reproduced in Fig. 7b, but with respect to the new nondimensional parameter $\tau$. All curves merge at $\tau=1$ where zero hysteresis is attained, but are significantly dispersed at high velocities where $\tau \square 1$. Interestingly, this is opposite to $\omega$. There, Fig. 7a, all curves merge at maximum hysteresis where $\omega \square 1$, and are significantly dispersed for low velocities, i.e. $\omega \square 1$. This can be intuitively explained as follows. For a prescribed $\hat{\beta} \hat{B}_{M}, \tau$ is proportional to $\omega$, thus is a pure measure of rate. However, unlike $\omega$, the magnitude of $\tau$ depends also on the height of the barrier. In this sense, an increase in $\hat{\beta} \hat{B}_{M}$ is equivalent to an exponential 
increase in rate. Keeping in mind that all curves merge when $\omega \square 1$, this influence of $\hat{\beta} \hat{B}_{M}$ leads to the observed dispersion at high $\tau$ values. On the other hand, equilibrium (or zero hysteresis) is reached when successful escapes over energy barriers are highly probable. Recalling that $\tau$ describes the number of successful escapes over the typical barrier, $\hat{\beta} \hat{B}_{M}$, explains why all curves merge at $\tau=1$.

In summary, $\omega$ and $\tau$ are both non-dimensional rates, but while $\omega$ measures rate with respect to the attempt rate $r_{0}, \tau$ measures it with respect to the rate of successful escapes over a typical barrier. Consequently, $\omega>1$ defines the range of maximum hysteresis (or high rates), while $\tau<1$ the range of quasi-equilibrium (or low rates). Still, none of these two seemingly natural choices of rate sets a universal scale that uniquely defines the range of low rates, high rates, and intermediate rates. A different approach is to define the ratio between typical energies rather than between time scales. Using expression (4.3), $\hat{\beta} \hat{B}_{M}=\ln \left(\frac{1}{2} N \cdot t_{M} / t_{0}\right)$ can be thought of as the (nondimensional) energy barrier associated with the time scale $t_{M}$. Similarly, we define the energy scale associated with $t_{c}$ as

$$
\hat{\beta} \hat{B}_{c}=\ln \left(\frac{1}{2} N \cdot t_{c} / t_{0}\right) .
$$

When the data are plotted against the new parameter

$$
P_{\omega} \equiv e^{-\hat{B}_{c} / \hat{B}_{M}}=(2 \omega / N)^{1 / \hat{\beta} \hat{B}_{M}},
$$

all sets of data collapse to a single curve (Fig. 7c). Note that $\tau=1$, which defines equilibrium, is equivalent to $P_{\omega}=e^{-1}$, while maximum hysteresis, associated with $\omega \square 1^{\dagger}$, corresponds with $P_{\omega}=1$. It is therefore evident that the new parameter $P_{\omega}$ well reflects the competition between rate, temperature, and energy barriers underlying the dynamics of the chain, and enables physical interpretation. In addition, $P_{\omega}$ sets a universal scale for rates with the following regions: (I) quasi-equilibrium ( $\left.P_{\omega}<e^{-1}\right)$, (II) stochastic response dominated by thermal fluctuations $\left(e^{-1}<P_{\omega}<1\right)$, and (III) maximum hysteresis $\left(P_{\omega}>1\right)$.

\footnotetext{
${ }^{\dagger}$ The keen reader might have noticed that all sets of data merge at $P_{\omega}=1$, see Fig. 7c, while at $\omega \approx 1$, see Fig. 7a. Eq. (4.7) indicates that $P_{\omega}=1$ corresponds to $\omega=N / 2$. Indeed, in Fig. 7a, all sets of data merge at $\omega=5$.
} 
Eq. (4.7), shows that $P_{\omega}$ has a mathematical structure similar to Boltzmann's factor, with $\hat{B}_{M}$ assuming the role of energy scale for $\hat{B}_{C}$. Alternatively, $P_{\omega}$ can roughly be thought of as a non-dimensional measure of rate, which reflects the probability of a system to remain in a meta-stable configuration during the experiment time scale $t_{c}$. Slower experiments (longer $t_{c}$, higher $\hat{B}_{c}$ ) yield lower probability, thus the system is more likely to switch configuration. The strength of this new parameter is further demonstrated in Fig. 8, showing the transition stress for a wide range of parametric values, with all data points lying on a single curve. This fundamentally important result signifies that although the system dynamics is complexly governed by a large set of parameters, this intricate behavior can be captured by a single nondimensional quantity, $P_{\omega}$.

\subsection{Dispersion of the transition stress}

In the previous section we have shown that the average transition stress can be described by means of a single parameter $P_{\omega}$. We note however, that this description does not provide a complete picture of the behavior. In particular, the dispersion of the transition force is of practical importance. To this end, we turn to studying the probability distribution function of the transition stress.

The cumulative number (fraction) of switching events from configuration $n$ to $n+1$ in the time interval $[0, \hat{t}]$ is

$$
P_{n}^{+}(\hat{t})=\int_{0}^{\hat{t}} I_{n}^{+}\left(\hat{t}^{\prime}\right) d \hat{t}^{\prime} .
$$

Since strain is an injective function of time and $\hat{\sigma}_{n}$ is an injective function of strain, $P_{n}^{+}(\hat{t})$ can be expressed in terms of the stress $\hat{\sigma}_{n}$ rather than time. Hence,

$$
f_{\sigma}=\frac{d P_{n}^{+}}{d \hat{\sigma}_{n}}
$$

is the probability density of the transition stress from configuration $n$ to $n+1$. From (2.1) and (2.12) we identify $I_{n}^{+}=(N-n) \exp \left(-\hat{\beta} \hat{B}_{n}^{+}\right) P_{n} / \omega$, thus

$$
f_{\sigma}=\frac{1}{\omega} \frac{N}{\phi \hat{K}_{n}}(N-n) \mathrm{e}^{-\hat{\beta} \hat{B}_{n}^{+}} P_{n}
$$


where relations $\frac{d P_{n}^{+}}{d \hat{\sigma}_{n}}=\frac{d P_{n}^{+}}{d \hat{t}} \frac{d \hat{t}}{d \hat{\varepsilon}} \frac{d \hat{\varepsilon}}{d \hat{\sigma}_{n}}, \hat{t}=\frac{t}{t_{c}}=N \hat{\varepsilon}$, and $d \hat{\sigma}_{n} / d \hat{\varepsilon}=\phi \hat{K}_{n}$ have been used. Note that the dependency of $f_{\sigma}$ with stress (or time) comes from the expression $\mathrm{e}^{-\hat{\beta} \hat{B}_{n}^{+}} P_{n}$, while the prefactor is simply a scaling factor which guarantees that the integral of $f_{\sigma}$ over the entire range is one.

Fig. 9a illustrates the probability density function $f_{\sigma}$ for various rates and relative barriers. The red (left) line shows $f_{\sigma}$ for $P_{\omega}=0.47$. The two black lines show $f_{\sigma}$ for higher values of $P_{\omega}$, realized by increasing $\omega$ while keeping the barriers (or equivalently temperature) unchanged. As expected, higher values of $P_{\omega}$ correspond to higher average transition stresses, manifested by the shift of the curves to the right, towards higher stresses. Note that, here, the dispersion increases as well. The two blue lines show $f_{\sigma}$ for the same values of $P_{\omega}$ as the black lines, however, this time the increase in $P_{\omega}$ is realized by increasing the relative barrier while keeping $\omega$ unchanged. Again, the average transition stress shifts to the right towards higher stresses and coincides with that of the black lines. However, now the dispersion decreases, contrary to the previous case. We therefore conclude that although the average transition stress directly depends on $P_{\omega}$, the dispersion of the transition stress does not. In addition, while the average transition stress is a monotonously increasing function of both $\omega$ and $\hat{\beta} \hat{B}_{M}, \omega$ and $\hat{\beta} \hat{B}_{M}$ have an opposite influence on the dispersion of the transition stress, see Fig. 9b,c. This can be understood by noting that increasing $\omega$ is equivalent to decreasing the frequency of attempts (number of attempts at each level of barrier) since $\omega \square r_{0}^{-1}$. Therefore, the dispersion increases with $\omega$. On the other hand, increasing $\hat{\beta} \hat{B}_{M}$ effectively decreases the magnitude of the energy disturbances. Clearly, a narrower distribution of disturbances leads to a narrower distribution of the transition force.

\subsection{The difference between the Maxwell and equilibrium paths}

The Maxwell and equilibrium paths are similar in the sense that both do not involve hysteresis, but they are not identical. In fact, the Maxwell path emerges as the zero temperature limit of the equilibrium response. In this limit, the statistical mechanics 
reduces to global energy minimization. Two main features distinguish between the equilibrium and the Maxwell stress-strain relations, as illustrated in Fig. 10: (i) the equilibrium path has a "tilt" (asymmetry), (ii) the magnitude of the saw-teeth is smaller in the equilibrium path. These differences stem from the fact that the Maxwell path involves exactly one configuration, the one corresponding to the lowest energy, at all times. On the other hand, the equilibrium path involves several configurations associated with multiple local minima that exist at each overall strain. Therefore, Maxwell's stress-strain relation includes jumps in stress associated with abrupt changes of configuration, resulting a sharp saw-tooth pattern. On the other hand, the equilibrium path experiences a continuous change of the distribution $P_{n}$, leading to a smooth (yet non-monotonous) stress-strain relation.

Our results regarding the difference between the Maxwell and equilibrium paths are not new. Yet, we present it here as a reminder that although the equilibrium path involves no hysteresis it is not the global minimizer. A detailed analysis of the equilibrium response and its zero-temperature limit is found in (Efendiev and Truskinovsky, 2010). There, the exact statistical sum (partition function) was computed for a bistable chain having bistable elements with different moduli at each well $(\lambda \neq 1)$, and statistical averaging was performed over the whole ensemble. The analysis here, on the other hand, is based on Kramers theory which only accounts for local minima and saddle points. This approach, pioneered by I. Muller in the context of materials (Achenbach and Muller, 1982; Muller and Seelecke, 2001) and by G.I. Bell and E. Evans in the context of molecular bonds (Bell, 1978; Evans, 2001; Evans and Ritchie, 1999), significantly simplifies the analysis, but is only suitable for systems with high barriers (deep wells). As discussed in Section 2, it is easy to verify that the equilibrium solution of (2.12) reduces to a distribution $P_{n}$ that is equal to Boltzmann distribution. The corresponding equilibrium stress-strain relation well approximates the actual equilibrium response for systems with high barriers. However, if barriers are low or if higher accuracy is required, one must turn to the more involved, but exact, computation presented in (Efendiev and Truskinovsky, 2010).

Finally, Fig. 10b demonstrates how several configurations are involved at equilibrium. We note that this feature is not limited to equilibrium, but also governs the behavior at moderate rates. 


\section{Understanding $P_{\omega}$ and further implications}

The notion of $P_{\omega}$ does not arise naturally from the non-dimensional formulation of the problem (2.12). In fact, the seemingly-natural candidates $\omega$ and $\tau$, which describe ratios of relevant time-scales, fail to provide a universal scale for rates. The reason is that $\omega$ is the relevant scale for extremely high rates while $\tau$ for extremely low rates. The definition of $P_{\omega}$, which involves a non-intuitive ratio between energy barriers, reconciles this discord. To better understand the role of $P_{\omega}$, it is useful to turn to a much simpler "toy" problem of a single irreversible energy barrier. This problem has been considered before, mainly in the context of irreversible rupture of inter-molecular bonds, e.g. (Dudko et al., 2006; Evans and Ritchie, 1999; Garg, 1995), and involves a single energy well with a single (irreversible) barrier, $B(t)$, which decreases monotonously from $B_{M}$ at $t=0$ to zero at $t=t_{c}^{s w}$ (upper index "sw" indicates "single well”). In this case, our set of equations (2.12) reduces to a single equation:

$$
\frac{d P}{d \hat{t}}=-\frac{1}{\omega} e^{-\hat{\beta} \hat{B}(\hat{t})} P=-r(\hat{t}) P,
$$

where $P$ is the "survival” probability of the bond. Note that here the experiment time scale, which also appears in $\omega$, is set by $t_{c}^{s \omega}$ (and not by $t_{c}$ as in (2.12). This difference is further discussed below), thus $\hat{B}=0$ at $\hat{t}=1$. In order to gain further insight, it is instructive to introduce the following transformation of $\omega$ (Herrmann et al., 2012), see also (4.6):

$$
\hat{\beta} \hat{B}_{c} \equiv-\ln \omega \Rightarrow \omega=e^{-\hat{\beta} \hat{B}_{c}},
$$

Plugging definition (5.2) into (5.1) yields

$$
r=e^{-\hat{\beta}\left(\hat{B}(\hat{t})-\hat{B}_{c}\right)},
$$

Recall that $\hat{B}(\hat{t})$ decreases with time from $\hat{B}_{M}$ at $\hat{t}=0$ to zero at $\hat{t}=1$, while $\hat{B}_{c}$ is constant. Roughly speaking, as long as $\hat{B}(\hat{t})-\hat{B}_{c}>0, r$ is very small due to the exponential dependency, thus the flux over the barrier is very small, effectively zero. On the other hand, when $\hat{B}(\hat{t})-\hat{B}_{c}<0, r$ becomes very large and all the population 
quickly leaves the local well by overcoming the barrier. For $\hat{B}_{c}<0$, we have that $\hat{B}(\hat{t})-\hat{B}_{c}>0$ at all time. Thus, the system remains at the bottom of the well, and switching will occur only when the barrier vanishes completely, i.e. at $\bar{t}=1$. Note that

$$
\hat{B}_{c}<0 \Rightarrow-\ln \omega<0 \quad \Rightarrow \omega>1 \text {, }
$$

In other words, if $\omega>1$ we get the so-called maximum hysteresis path, with transition stress $\hat{\sigma}_{\max }$.

The other limit case is for $b>B_{M}$. Here, $B(\hat{t})-\mathrm{b}<0$ for all $t>0$. This means that immediately after the experiment begins, all the population quickly leaves the local minimum by overcoming the barrier. Note that

$$
\hat{B}_{c}>\hat{B}_{M} \Rightarrow-\ln \omega>\hat{\beta} \hat{B}_{M} \Rightarrow P_{\omega}<e^{-1}
$$

In other words, if $P_{\omega}<e^{-1}$ the system follows the equilibrium path.

In the case of $0<\hat{B}_{c}<\hat{B}_{M}, B(\hat{t})-\hat{B}_{c}$ is positive at first, but changes sign at some time. At this time, when $\hat{B}(\hat{t})-\hat{B}_{c}$ becomes negative, all the population quickly leaves the local minimum. This occurs at the time $\hat{t}^{t}$ that satisfies $B\left(\hat{t}^{t}\right)=\hat{B}_{c}$.

Consider now the rather general class of barriers having time dependency of the form

$$
\hat{\beta} \hat{B}(\hat{t})=\hat{\beta} \hat{B}_{M}(1-\hat{t})^{m} .
$$

Following the above, we have that the most probable transition time is

$$
\hat{t}^{t}=1-\left(\ln \left(P_{\omega}^{-1}\right)\right)^{1 / m}=1-\left(\hat{B}_{c} / \hat{B}_{M}\right)^{1 / m}
$$

Note that, above, we have taken the heuristic assumption that all the population remains in the local minimum as long as $\hat{B}(\hat{t})-\hat{B}_{c}>0$, and immediately leaves it once $\hat{B}(\hat{t})-\hat{B}_{c}>0$. This approach may provide intuition and rough approximation, but is not exact. To further understand this, let us consider the rigorous definition of the most probable transition time, $\hat{t}^{t}$, i.e. 


$$
\left.\frac{d^{2} P}{d \hat{t}^{2}}\right|_{\hat{t}=\hat{t}^{t}}=\left.0 \Rightarrow \frac{d}{d \hat{t}}(\hat{\beta} \hat{B}(\hat{t})) e^{\hat{\beta} \hat{B}(\hat{t})}\right|_{\hat{t}=\hat{t}^{t}}=-\frac{1}{\omega} .
$$

Next, by plugging (5.7) into (5.8) we find that the "shape" (time dependency) of the barrier that results in a transition time (5.7) takes the form

$$
\hat{\beta} \hat{B}(\hat{t})=\ln \left(1+\frac{1}{m}\left(-\hat{\beta} \hat{B}_{M}\right)^{-1 / m}\left(\Gamma\left(\frac{1}{m}\right)-\Gamma\left(\frac{1}{m},-\hat{\beta} \hat{B}_{M}(1-\hat{t})^{m}\right)\right)\right),
$$

where, $\Gamma(z)$ and $\Gamma(z, b)$ are the Euler and incomplete gamma functions, respectively, related by $\Gamma(z, 0)=\Gamma(z)$. One can be convinced with result (5.9) by inserting (5.9) directly into (5.8) and obtaining $\hat{t}^{t}$. It is easy to verify that the resulting transition time is exactly (5.7). Interestingly, for $\hat{\beta} \hat{B}_{M} \square 1$, relation (5.9) is well approximated by (5.6), as illustrated in Fig. 11a. This can also be shown by a somewhat different approach, i.e. by plugging (5.6) directly into (5.1) and deriving an asymptotic (small rates) approximation for the average transition time. One can show that the leading order of this approximation takes a form similar to that of (5.7) (Dudko et al., 2006; Garg, 1995).

Let us return now to a chain having multiple energy wells, and recall that relation (5.6) is for systems having a single well. In the context of a chain, $\hat{t}$ in (5.6) should measure the time relative to $\hat{t}_{\text {Maxwell }}$, i.e. the time at which the overall strain is equal to Maxwell's strain $\hat{\varepsilon}_{M}$. Accordingly, $\hat{t}^{t}$ in the left-hand-side of relation (5.7) should be replaced by $\bar{t}^{t}$, defined as

$$
\bar{t}^{t} \equiv \frac{\hat{t}^{t}-\hat{t}_{\text {Maxwell }}}{\hat{t}_{c}^{s w}-\hat{t}_{\text {Maxwell }}}
$$

Above, the denominator indicates the time it takes the local well (or alternatively the barrier) to vanish, measured from $\hat{t}_{\text {Maxwell }}$. Accordingly, $0 \leq \bar{t}^{t} \leq 1$. Since the chain is pulled at constant strain rate and the energy barriers of the tri-linear chain are quadratic functions of strain (see Section 3), the barriers take the form (5.6) with $m=2$. Further, for a prescribed configuration, the stress of the tri-linear chain is linear with strain, hence linear with time. Since transition stress is measured relatively to Maxwell's stress (see Section 4.1), i.e. it is zero at $\hat{t}=\hat{t}_{\text {Maxwell }}$, we have that 


$$
\bar{t}^{t}=\bar{\sigma}^{t}
$$

Combining (4.1), (5.7), and (5.11), we conclude that

$$
\bar{\sigma}^{t}=1-\left(\ln \left(P_{\omega}^{-1}\right)\right)^{1 / 2}
$$

for the tri-linear chain. With the experiment time scale set by $t_{c}$ (3.8), and not by $t_{c}^{s w}$ as in previous studies (this crucial point is further discussed in the next paragraph), approximation (5.12) is remarkably consistent with the results of our simulations, as illustrated by the solid line in Fig. 7c and Fig. 8. This result is striking since approximation (5.12) is subject to three important assumptions: (i) a single energy well ( $N=1$ ), (ii) irreversible barrier, and (iii) small rates (Garg, 1995). All three assumptions are clearly violated in the case of a bistable chain. Specifically, we have shown in our simulations that several configurations are simultaneously involved (see Fig.10b for example), reversible transitions indeed take place (these are also responsible for the existence of quasi-equilibrium at very low rates), and finally, relation (5.12) provides an excellent approximation for the entire range of rates, from quasi-equilibrium to maximum hysteresis.

Note that since $P_{\omega}$ strongly depends on the choice of the experiment time scale $t_{c}$, a proper definition of $t_{c}$ is crucial for the accuracy of approximation (5.12). Definition (3.8) sets the experiment time scale to be the typical time the chain spends in a configuration before switching to the next. In particular, $t_{c}=A a / v=L \varepsilon_{c} / v$ where $\varepsilon_{c}=A \hat{\varepsilon}_{c}=A / N$ is the width of Maxwell's-path saw tooth in the stress-strain response (see Fig. 6). On the other hand, motivated by single barrier models, previous studies have adopted the seemingly natural choice for the experiment time scale, $t_{c}^{s w}$. $t_{c}^{s w}$ describes the lifetime of an energy well, i.e. the typical time the local minima exists until the barrier separating it from the next configuration vanishes. Clearly, $t_{c}^{s w} \neq t_{c}$. A geometric interpretation demonstrating this difference in terms of the strains $\varepsilon_{c}=t_{c} v / L$ and $\varepsilon_{c}^{s w}=t_{c}^{s w} v / L$ is shown in Fig. 6. Here, $\varepsilon_{c}^{s w}$ is the range of strain for which the meta-stable configuration exists. The fundamental difference between the two definitions is revealed when considering the dependence of these time scales with $N$. While $\hat{\varepsilon}_{c}^{s w}$ increases with $N, \hat{\varepsilon}_{c}$ decreases with $N$. Note that the two timescales coincide in the case of a single barrier. Also, for very high barriers, 
$P_{\omega}$ is indifferent to the difference between the two. Thus, it is instructive to examine the consequences of the opposite dependency of the two timescales with $N$ for systems with relatively small barriers. Fig. 12 compares between the transition stresses obtained for the two choices, $t_{c}$ and $t_{c}^{s w}$, in the case of $\hat{\beta} \hat{B}_{M} \approx 10$. As the number of elements in the chain increases, the results with $t_{c}$ approach the analytical approximation, while the accuracy with $t_{c}^{s w}$ significantly reduces. These results, combined with the fact that the definitions of $t_{c}$ and $t_{c}^{s w}$ coincide for a single barrier, suggest that $t_{c}$ is the proper experiment time scale, suitable for systems having multiple (or single) metastable configurations.

The above conclusions have important implications to the rate-dependent response of bistable chains, not necessarily composed of tri-linear elements. In Section 2, we have shown that the response of the system merely depends on the time-dependent energy barriers. With tri-linear elements, these were quadratic functions of time, i.e. $m=2$. If a chain is composed of other elements, the analysis is identical except that the dependence of the barriers with time is different. For example, a chain composed of bistable elements with cubic stress-strain relation that is subjected to a constant strain rate exhibits energy barriers with $m=4$ (Puglisi and Truskinovsky, 2000). Roughly, (5.6) can be used to approximate the barriers of almost any type of bistable elements, even if their stress-strain relation is not strictly polynomial. A practical example is a chain of bistable elements, each consisting of a hydrogen bond connected in series to a worm-like-chain. This configuration is commonly used to model macromolecules with compactly folded domains. In this case, energy barriers can be approximated with $m=0.57$. Our results above suggest that the average transition time of such chains can be well approximated by (5.7) with $\bar{t}^{t}$ replacing $\hat{t}^{t}$ there. Importantly, no matter what the exact functional relation of the barriers is, the three ranges of rate remain (I) $P_{\omega}<e^{-1}$, (II) $e^{-1}<P_{\omega}<1$, and (III) $P_{\omega}>1$, see also Fig. 11b. The only difference is the "shape" of the curve describing the dependency between transition time and $P_{\omega}$ in the intermediate range, which directly depends on $m$. Note that once the average transition time is found, it can be used to provide an estimate for the average strain or average stress by a straight forward transformation that involves the externally-controlled ramp rate (of either stress or strain) and the 
specific stress-strain relation of the elements in the chain. This is true not only for length-control conditions (hard-device/ Helmholtz ensemble) but also to load-control conditions (soft-device/ Gibbs Ensemble). Although this approach unifies the analysis of length-control and force-control conditions, one must notice that the response of a chain may significantly differ between these two loading types. This stems from two main sources. Firstly, two identical chains, one is length-controlled and the other is force-controlled, exhibit different barriers even at the same level of stress. This is illustrated in Fig. 13a for a tri-linear chain. Here, the force-control barrier from configuration $n=0$ to $n=1$ is illustrated by the shaded area, while the length-control barrier by the hatched area. Secondly, Maxwell's stress, which sets the reference stress for measuring the transition stress, may differ significantly between lengthcontrol and force-control conditions, see Fig. 13b. These important features have traditionally been overlooked in previous studies or ignored by assuming that the chain (or macromolecule) is attached to a very soft spring. Thus, enforcing soft-device conditions even if overall length is controlled.

Finally, we emphasize that the current model, and consequently the results presented herein, are limited by the inherent assumption that the system is adjusted instantaneously to the time-dependent energy landscape. This implies separation of time scales between the stochastic dynamics associated with switching between metastable configurations and the dynamics within a metastable configuration (Herrmann et al., 2012). Consequently, the contribution of the damping force (viscosity), $\eta v$, has been neglected. Clearly, this assumption is valid as long as the "rate condition” is satisfied:

$$
\frac{\eta v}{\Delta \sigma} \square 1
$$

Using relations (2.6), (2.13), and (3.8) we find that

$$
\frac{\eta v}{\Delta \sigma}=\frac{1}{2 \pi r_{0} L} \frac{k}{\sqrt{|1-\phi|}} \frac{A a}{t_{c}} \frac{1}{\Delta \sigma}=\frac{1}{2 \pi} \frac{\phi}{\sqrt{|1-\phi|}} \frac{\omega}{N} .
$$

Using (5.14), condition (5.13) may be rewritten:

$$
\frac{1}{2 \pi} \frac{\phi}{\sqrt{|1-\phi|}} \frac{\omega}{N}<d
$$


where $d$ is some small number. For practical purposes, the "engineering rule" of $d=5 \%$ may be considered. Using the definition of $P_{\omega},(4.7)$, the rate condition is

$$
P_{\omega}<\left(d \frac{4 \pi}{\phi} \sqrt{|1-\phi|}\right)^{1 / \beta B_{M}} .
$$

For the case of $\beta B_{M} \square 1$ the rate condition reduces to $P_{\omega}<1$. For example, for $\beta B_{M}=100$ and $\phi=1.25$, (5.16) implies that $P_{\omega}<0.99$, while for $\beta B_{M}=30$, $P_{\omega}<0.96$. In other words, $P_{\omega}$ also offers a practical perspective on the significance of the damping force. If $P_{\omega}>1$, the hysteresis is damping-dominated, and the contribution of viscos force, $\eta v$, must be accounted for.

\section{Summary and Conclusions}

We have studied the rate dependent response of a bistable chain subjected to thermal fluctuations. The study is motivated by the fact that the behavior of this model system is prototypical to a wide range of nonlinear processes in materials physics, biology and chemistry. Although this model-system is essentially 1D, its energy landscape is extremely wiggly and depends on a large number of degrees of freedom. In the case of high energy barriers, this complexity can be reduced to a $1 \mathrm{D}$ energy landscape defined by means of a reaction path. This path typically crosses the lowest saddle points that separate between metastable configurations. This simplification is also a practical approach in the sense that mapping the entire energy landscape of real systems is extremely hard. In this sense, although the bistable chain model is essentially $1 \mathrm{D}$, it may be applied to understanding the behavior of more general systems having rugged energy landscape. The dynamics of the chain is governed by a large set of parameters, such as number of elements, overall stiffness, temperature, and rate, in a complex way. Depending on these parameters, the response of the system can be stochastic or deterministic. In particular, at extremely low rates, the system operates at quasi-equilibrium; at moderate rates thermal noise dominates the behavior, resulting in a stochastic response; and at sufficiently high rates, the system has no time to explore its energy landscape, so stochastic fluctuations become irrelevant and the response is deterministic. In the first region (low rates), the system 
exhibits no hysteresis and transition stresses are the lowest. At intermediate rates, hysteresis mainly stems from transitions from high-energy to low-energy configurations, and the magnitudes of the average transition stress increases nonlinearly with rate. Finally, at very high rates, hysteresis is dominated by standard damping mechanism.

Based on a large set of numerical simulations, we have demonstrated that the rates defining each region may differ significantly depending on the specific parameters of the system. However, we found that fundamental characteristics of this response, namely the average transition stress, can be captured by a single non-dimensional parameter, $P_{\omega}$, which sets a universal scale that uniquely determines whether a rate is fast, slow or intermediate. The importance of $P_{\omega}$ is threefold: (i) it facilitates predicting fundamental behavioral features, namely average transition stress and consequently hysteresis, (ii) it provides a simple and direct benchmark for rates, and (iii) it unifies the response of a wide range of systems. In order to determine $P_{\omega}$ one only needs to know the ratio between the two energy scales $B_{c}$ and $B_{M}$. While $B_{M}$, the barrier on the Maxwell's path, has a direct interpretation of a typical energy scale, $B_{c}$ is not a real barrier. It is a transformation of the experiment time-scale, $t_{c}$, to an energy-scale. In other words, $B_{c}$ is the height of a pseudo barrier associated with “escape” rate $1 / t_{c}$.

We emphasize that the competence of $P_{\omega}$ as a universal scale relies heavily on proper definitions of the transition rate and of the experimental time scale $t_{c}$. In particular, transition stress must be measured relative to the Maxwell's stress. In simple models involving a single energy well with irreversible barrier, the notion of Maxwell's path does not exist and transition stress is measured relative to the arbitrary starting point of zero stress. This arbitrary choice of reference stress is not appropriate for systems that include multiple metastable configurations. Furthermore, we have shown that $t_{c}$ must reflect the typical time the system maintains a configuration. Previous studies on the other hand, have traditionally adopted the typical lifetime of an energy well, $t_{c}^{s w}$, as the experiment time scale. This seemingly natural choice, motivated from single-barrier models, is fundamentally different from 
$t_{c}$. This difference is clearly revealed when considering the dependence of these time scales with $N$. While $\varepsilon_{c}^{s b}=t_{c}^{s b} v / L$ increases with $N, \varepsilon_{c}=t_{c} v / L$ decreases with $N$. Based on the above insights, we have shown that the analysis can be naturally extended to a wide range of bistable chains, not necessarily tri-linear, and to any loading conditions. Still, one must note that different loading conditions correspond to different energy barriers, and that these barriers govern the response. In particular, the barriers of two identical chains subjected to length control and force control conditions may differ significantly, even for the same level of force. Moreover, Maxwell's stress, which sets the reference stress for measuring the transition stress, may differ substantially in length control and force control conditions. These features must be accounted for in order to correctly reflect actual loading conditions.

\section{Acknowledgements:}

This work was supported by the Israel Science Foundation grant ISF 724/14 to S.G., and by the National Science Foundation grant MCB-1411884 to O.K.D. 


\section{References}

Achenbach, M., Muller, I., 1982. A Model for Shape Memory. J Phys-Paris 43, 163-167.

Balk, A.M., Cherkaev, A.V., Slepyan, L.I., 2001. Dynamics of chains with non-monotone stress-strain relations. I. Model and numerical experiments. Journal of the Mechanics and Physics of Solids 49, 131-148.

Bell, G., 1978. Models for the specific adhesion of cells to cells. Science 200, 618-627.

Benichou, I., Faran, E., Shilo, D., Givli, S., 2013. Application of a bi-stable chain model for the analysis of jerky twin boundary motion in NiMnGa. Applied Physics Letters 102.

Benichou, I., Givli, S., 2011. The hidden ingenuity in titin structure. Applied Physics Letters 98, 091904-091904-091903.

Benichou, I., Givli, S., 2013. Structures undergoing discrete phase transformation. Journal of the Mechanics and Physics of Solids 61, 94-113.

Benichou, I., Givli, S., 2015. Rate Dependent Response of Nanoscale Structures Having a Multiwell Energy Landscape. Physical Review Letters 114, 095504.

Bonilla, L.L., Carpio, A., Prados, A., 2015. Theory of force-extension curves for modular proteins and DNA hairpins. Phys Rev E 91.

Bosaeus, N., El-Sagheer, A.H., Brown, T., Smith, S.B., Åkerman, B., Bustamante, C., Nordén, B., 2012. Tension induces a base-paired overstretched DNA conformation. Proceedings of the National Academy of Sciences 109, 15179-15184.

Braides, A., Dal Maso, G., Garroni, A., 1999. Variational formulation of softening phenomena in fracture mechanics: The one-dimensional case. Archive for Rational Mechanics and Analysis 146, 23-58.

Brown, A.E.X., Litvinov, R.I., Discher, D.E., Purohit, P.K., Weisel, J.W., 2009. Multiscale Mechanics of Fibrin Polymer: Gel Stretching with Protein Unfolding and Loss of Water. Science 325, 741-744.

Brown, A.E.X., Litvinov, R.I., Discher, D.E., Weisel, J.W., 2007. Forced unfolding of coiled-coils in fibrinogen by single-molecule AFM. Biophysical Journal 92, L39-L41.

Cherkaev, A., Cherkaev, E., Slepyan, L., 2005. Transition waves in bistable structures. I. Delocalization of damage. Journal of the Mechanics and Physics of Solids 53, 383405.

Chyan, C.-L., Lin, F.-C., Peng, H., Yuan, J.-M., Chang, C.-H., Lin, S.-H., Yang, G., 2004a. Reversible mechanical unfolding of single ubiquitin molecules. Biophysical Journal 87, 3995-4006.

Chyan, C.L., Lin, F.C., Peng, H., Yuan, J.M., Chang, C.H., Lin, S.H., Yang, G., 2004b. Reversible mechanical unfolding of single ubiquitin molecules. Biophysical Journal 87, 39954006.

Cohen, T., Givli, S., 2014. Dynamics of a discrete chain of bi-stable elements: A biomimetic shock absorbing mechanism. Journal of the Mechanics and Physics of Solids 64, 426439.

Dan Mordehai, S.-W.L., Björn Backes, David J. Srolovitz, William D. Nix, Eugen Rabkin, 2011. Size effect in compression of single-crystal gold microparticles. Acta Materialia 59, 5202-5215.

Dreyer, W., Guhlke, C., Herrmann, M., 2011. Hysteresis and phase transition in many-particle storage systems. Continuum Mechanics and Thermodynamics 23, 211-231.

Dreyer, W., Jamnik, J., Guhlke, C., Huth, R., Moskon, J., Gaberscek, M., 2010. The thermodynamic origin of hysteresis in insertion batteries. Nature Materials 9 , 448453.

Dudko, O.K., 2015. Decoding the mechanical fingerprints of biomolecules. Quarterly reviews of biophysics, 1-14. 
Dudko, O.K., Hummer, G., Szabo, A., 2006. Intrinsic Rates and Activation Free Energies from Single-Molecule Pulling Experiments. Physical Review Letters 96, 108101.

Efendiev, Y.R., Truskinovsky, L., 2010. Thermalization of a driven bi-stable FPU chain. Continuum Mech. Thermodyn 22, 679-698.

Evans, E., 2001. Probing the relation between force-lifetime-and chemistry in single molecular bonds. Annual review of biophysics and biomolecular structure 30, 105128.

Evans, E., Ritchie, K., 1997. Dynamic strength of molecular adhesion bonds. Biophysical Journal 72, 1541-1555.

Evans, E., Ritchie, K., 1999. Strength of a weak bond connecting flexible polymer chains. Biophysical Journal 76, 2439-2447.

Fedelich, B., Zanzotto, G., 1992. Hysteresis in discrete systems of possibly interacting elements with a double-well energy. Journal of Nonlinear Science 2, 319-342.

Friddle, R.W., Noy, A., De Yoreo, J.J., 2012. Interpreting the widespread nonlinear force spectra of intermolecular bonds. Proceedings of the National Academy of Sciences 109, 13573-13578.

Garg, A., 1995. Escape-field distribution for escape from a metastable potential well subject to a steadily increasing bias field. Physical Review B 51, 15592-15595.

Givli, S., 2010. Towards multi-scale modeling of muscle fibers with sarcomere nonuniformities. Journal of Theoretical Biology 264, 882-892.

Givli, S., Bhattacharya, K., 2009. A coarse-grained model of the myofibril: Overall dynamics and the evolution of sarcomere non-uniformities. Journal of the Mechanics and Physics of Solids 57, 221-243.

Gross, P., Laurens, N., Oddershede, L.B., Bockelmann, U., Peterman, E.J.G., Wuite, G.J.L., 2011. Quantifying how DNA stretches, melts and changes twist under tension. Nat Phys 7, 731-736.

Hänggi, P., Talkner, P., Borkovec, M., 1990. Reaction-rate theory: fifty years after Kramers. Reviews of Modern Physics 62, 251.

Herrmann, M., Niethammer, B., Velazquez, J.J.L., 2012. Kramers and Non-Kramers Phase Transitions in Many-Particle Systems with Dynamical Constraint. Multiscale Model $\operatorname{Sim} 10,818-852$.

Huo, Y., Müller, I., 1993. Nonequilibrium thermodynamics of pseudoelasticity. Continuum Mech. Thermodyn 5, 163-204.

Jennings, A.T., Li, J., Greer, J.R., 2011. Emergence of strain-rate sensitivity in Cu nanopillars: Transition from dislocation multiplication to dislocation nucleation. Acta materialia 59, 5627-5637.

Keller, D., Swigon, D., Bustamante, C., 2003. Relating Single-Molecule Measurements to Thermodynamics. Biophysical Journal 84, 733-738.

Kellermayer, M.S.Z., Smith, S.B., Granzier, H.L., Bustamante, C., 1997. Folding-Unfolding Transitions in Single Titin Molecules Characterized with Laser Tweezers. Science 276, 1112-1116.

Kinderlehrer, D., Ma, L., 1994. COMPUTATIONAL HYSTERESIS IN MODELING MAGNETIC SYSTEMS. leee Transactions on Magnetics 30, 4380-4382.

King, G.A., Gross, P., Bockelmann, U., Modesti, M., Wuite, G.J.L., Peterman, E.J.G., 2013. Revealing the competition between peeled ssDNA, melting bubbles, and S-DNA during DNA overstretching using fluorescence microscopy. Proceedings of the National Academy of Sciences 110, 3859-3864.

King, W.T., Su, M.H., Yang, G.L., 2010. Monte Carlo simulation of mechanical unfolding of proteins based on a simple two-state model. International Journal of Biological Macromolecules 46, 159-166. 
Kunz, A., Pathak, S., Greer, J.R., 2011. Size effects in Al nanopillars: Single crystalline vs. bicrystalline. Acta materialia 59, 4416-4424.

Labeit, D., Watanabe, K., Witt, C., Fujita, H., Wu, Y., Lahmers, S., Funck, T., Labeit, S., Granzier, H., 2003. Calcium-dependent molecular spring elements in the giant protein titin. Proceedings of the National Academy of Sciences 100, 13716-13721.

Linke, W.A., Grutzner, A., 2008. Pulling single molecules of titin by AFM - recent advances and physiological implications. Pflugers Archiv European Journal of Physiology 456, 101-115.

Maaß, R., Meza, L., Gan, B., Tin, S., Greer, J.R., 2012. Ultrahigh Strength of Dislocation-Free Ni3Al Nanocubes. Small 8, 1869-1875.

Manca, F., Giordano, S., Palla, P.L., Cleri, F., 2014. On the equivalence of thermodynamics ensembles for flexible polymer chains. Physica A: Statistical Mechanics and its Applications 395, 154-170.

Manca, F., Giordano, S., Palla, P.L., Cleri, F., Colombo, L., 2012a. Theory and Monte Carlo simulations for the stretching of flexible and semiflexible single polymer chains under external fields. Journal of Chemical Physics 137.

Manca, F., Giordano, S., Palla, P.L., Cleri, F., Colombo, L., 2013. Two-state theory of singlemolecule stretching experiments. Physical Review E 87.

Manca, F., Giordano, S., Palla, P.L., Zucca, R., Cleri, F., Colombo, L., 2012b. Elasticity of flexible and semiflexible polymers with extensible bonds in the Gibbs and Helmholtz ensembles. The Journal of Chemical Physics 136, -.

Mirny, L., Shakhnovich, E., 2001. Protein folding theory: From lattice to all-atom models. Annual Review of Biophysics and Biomolecular Structure 30, 361-396.

Muller, I., Seelecke, S., 2001. Thermodynamic aspects of shape memory alloys. Math Comput Model 34, 1307-1355.

Müller, I., Villaggio, P., 1977. A model for an elastic-plastic body. Archive for Rational Mechanics and Analysis 65, 25-46.

Muller, I., Xu, H., 1991. On the pseudo-elastic hysteresis. Acta Metallurgica et Materialia 39, 263-271.

Oberhauser, A.F., 2001. Stepwise unfolding of titin under force-clamp atomic force microscopy. Proceedings of the National Academy of Sciences 98, 468-472.

Oberhauser, A.F., Badilla-Fernandez, C., Carrion-Vazquez, M., Fernandez, J.M., 2002. The mechanical hierarchies of fibronectin observed with single-molecule AFM. Journal of molecular biology 319, 433-447.

Oberhauser, A.F., Hansma, P.K., Carrion-Vazquez, M., Fernandez, J.M., 2001. Stepwise unfolding of titin under force-clamp atomic force microscopy. Proceedings of the National Academy of Sciences 98, 468-472.

Oberhauser, A.F., Marszalek, P.E., Erickson, H.P., Fernandez, J.M., 1998. The molecular elasticity of the extracellular matrix protein tenascin. Nature 393, 181-185.

Pattamatta, S., Elliott, R.S., Tadmor, E.B., 2014. Mapping the stochastic response of nanostructures. Proceedings of the National Academy of Sciences 111, E1678-E1686.

Plata, C.A., Cecconi, F., Chinappi, M., Prados, A., 2015. Understanding the dependence on the pulling speed of the unfolding pathway of proteins. J Stat Mech-Theory E.

Puglisi, G., Truskinovsky, L., 2000. Mechanics of a discrete chain with bi-stable elements. Journal of the Mechanics and Physics of Solids 48, 1-27.

Puglisi, G., Truskinovsky, L., 2002a. A mechanism of transformational plasticity. Continuum Mech. Thermodyn 14, 437-457.

Puglisi, G., Truskinovsky, L., 2002b. Rate independent hysteresis in a bi-stable chain. Journal of the Mechanics and Physics of Solids 50, 165-187. 
Purohit, P.K., Litvinov, R.I., Brown, A.E.X., Discher, D.E., Weisel, J.W., 2011. Protein unfolding accounts for the unusual mechanical behavior of fibrin networks. Acta Biomater. 7, 2374-2383.

Q.P. Sun, Y.J.H., 2008. A multiscale continuum model of the grain-size dependence of the stress hysteresis in shape memory alloy polycrystals. International Journal of Solids and Structures 45, 3868-3896.

Rico, F., Gonzalez, L., Casuso, I., Puig-Vidal, M., Scheuring, S., 2013. High-speed force spectroscopy unfolds titin at the velocity of molecular dynamics simulations. science 342, 741-743.

Rief, M., Gautel, M., Oesterhelt, F., Fernandez, J.M., Gaub, H.E., 1997. Reversible Unfolding of Individual Titin Immunoglobulin Domains by AFM. Science 276, 1109-1112.

Rief, M., Gautel, M., Schemmel, A., Gaub, H.E., 1998. The Mechanical Stability of Immunoglobulin and Fibronectin III Domains in the Muscle Protein Titin Measured by Atomic Force Microscopy. Biophysical Journal 75, 3008-3014.

Risken, H., 1984. Fokker-Planck Equation. Springer.

Ritwik Raj, P.K.P., 2011. hase boundaries as agents of structural change in macromolecules. Journal of the Mechanics and Physics of Solids 59, 2044-2069.

Rogers, R.C., Truskinovsky, L., 1997. Discretization and hysteresis. Physica B: Condensed Matter 233, 370-375.

Schlierf, M., Li, H., Fernandez, J.M., 2004. The unfolding kinetics of ubiquitin captured with single-molecule force-clamp techniques. Proceedings of the National Academy of Sciences of the United States of America 101, 7299-7304.

Schwaiger, I., Sattler, C., Hostetter, D.R., Rief, M., 2002. The myosin coiled-coil is a truly elastic protein structure. Nature Materials 1, 232-235.

Seelecke, S., Kim, S.-J., Ball, B., Smith, R., 2005. A rate-dependent two-dimensional free energy model for ferroelectric single crystals. Continuum Mech. Thermodyn 17, 337350.

Shulha, H., Foo, C.W.P., Kaplan, D.L., Tsukruk, V.V., 2006. Unfolding the multi-length scale domain structure of silk fibroin protein. Polymer 47, 5821-5830.

Singh, A.R., Giri, D., Kumar, S., 2011. Statistical mechanics of stretching of biopolymers. Journal of Statistical Mechanics: Theory and Experiment 2011, P05019.

Sinha, S., Samuel, J., 2005. Inequivalence of statistical ensembles in single molecule measurements. Physical Review E 71, 021104.

Slepyan, L.I., Troyankina, L.V., 1984. Fracture wave in a chain structure. J Appl Mech Tech Phys 25, 921-927.

Staple, D.B., Payne, S.H., Reddin, A.L.C., Kreuzer, H.J., 2009. Stretching and unfolding of multidomain biopolymers: a statistical mechanics theory of titin. Physical Biology 6.

Su, T.X., Purohit, P.K., 2009. Mechanics of forced unfolding of proteins. Acta Biomater. 5, 1855-1863.

Suess, D., Fidler, J., 2006. Miocromagnetic simulation of magnetic materials, in: Buschow, K.H.J. (Ed.), Handbook of magnetic materials. Elsevier, Amsterdam.

Truskinovsky, L., 1996. Fracture as a phase transition, in: Batra, R.C., Beatty, M.F. (Eds.), Contemporary research in the mechanics and mathematics of materials. CIMNE, Barcelona, pp. 322-332.

Tskhovrebova, L., Trinick, J., Sleep, J.A., Simmons, R.M., 1997. Elasticity and unfolding of single molecules of the giant muscle protein titin. Nature 387, 308-312.

Vainchtein, A., 2010. The role of spinodal region in the kinetics of lattice phase transitions. Journal of the Mechanics and Physics of Solids 58, 227-240.

Wang, Z.-J., Li, Q.-J., Shan, Z.-W., Li, J., Sun, J., Ma, E., 2012. Sample size effects on the large strain bursts in submicron aluminum pillars. Applied Physics Letters 100, -. 
Williams, P.M., Fowler, S.B., Best, R.B., Luis Toca-Herrera, J., Scott, K.A., Steward, A., Clarke, J., 2003. Hidden complexity in the mechanical properties of titin. Nature $422,446-$ 449.

Winkler, R.G., 2010. Equivalence of statistical ensembles in stretching single flexible polymers. Soft Matter 6, 6183-6191.

Zhao, Q., Purohit, P.K., 2014. Extracting a kinetic relation from the dynamics of a bistable chain. Modelling and Simulation in Materials Science and Engineering 22.

Zhurkov, S., 1965. Thermofluctuation mechanism of fracture. Int. J. Fract. Mech 1, 311-323. 


\section{Figures}

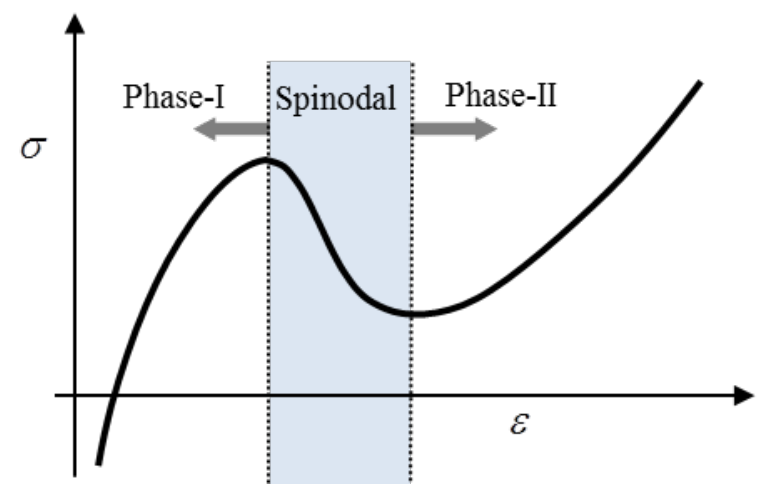

Figure 1: Stress-strain relation of a bistable element.

Cross-ref: Fig. 1

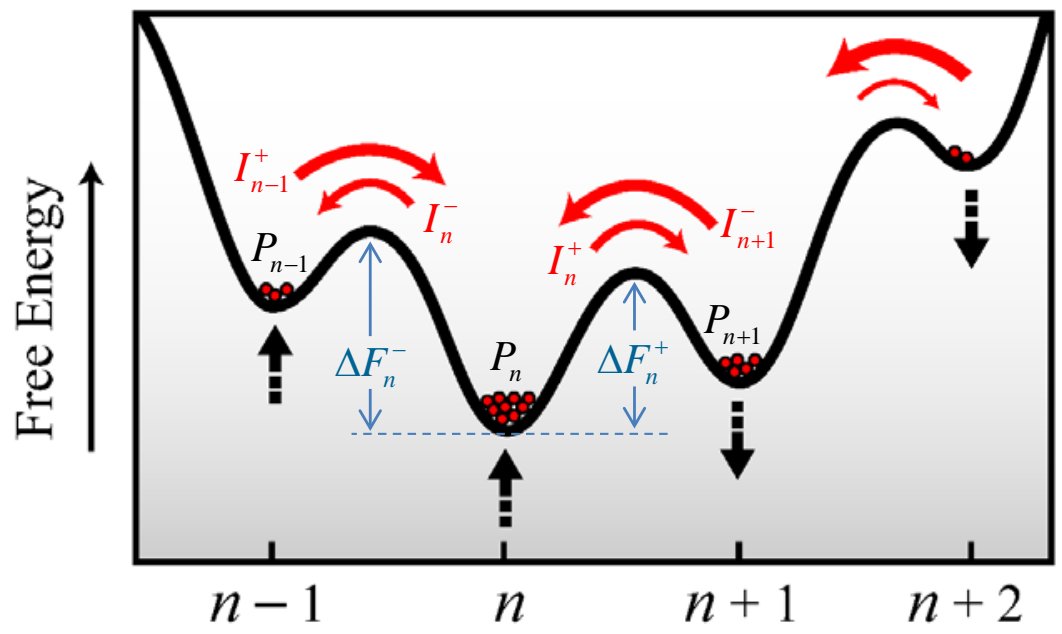

Figure 2: Schematic illustration of the simplified free-energy landscape of the chain at a prescribed overall strain. Overall strain is time dependent; hence, the barriers change in time. $\Delta F_{n}^{ \pm}$indicate the minimal energy barriers, or saddle points, separating between configuration $n$ and configurations $n \pm 1$.

Cross-ref: Fig. 2 


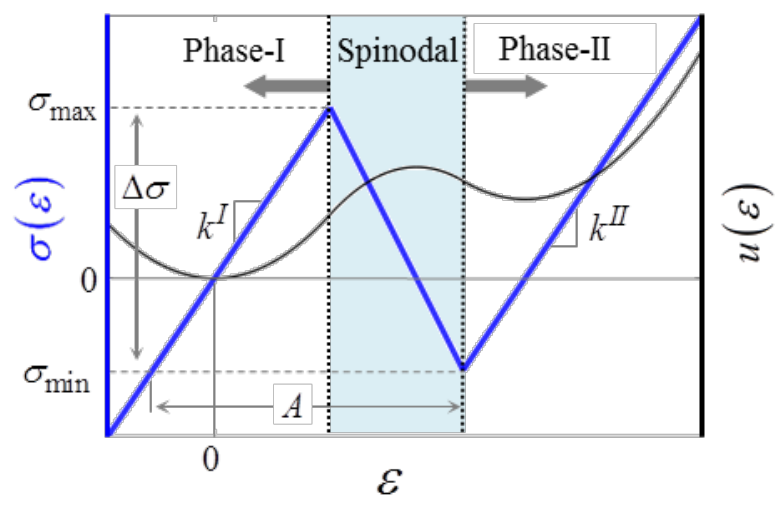

Figure 3: The tri-linear bistable element. Stress-strain relation (blue) and energy-strain relation (black). Shaded area marks the spinodal region.

Cross-ref: Fig. 3 


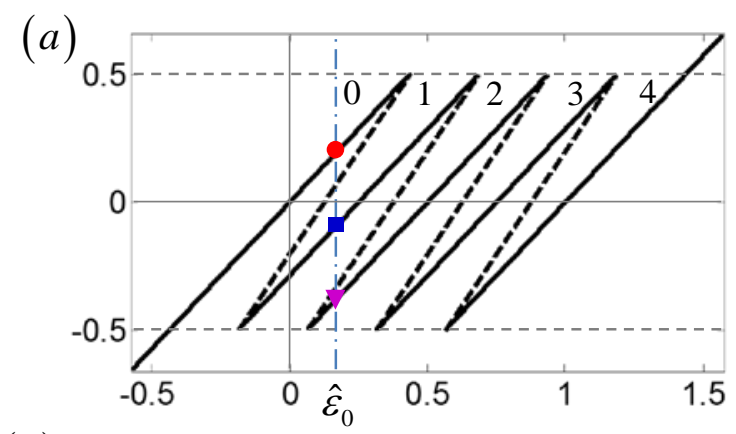

(b)

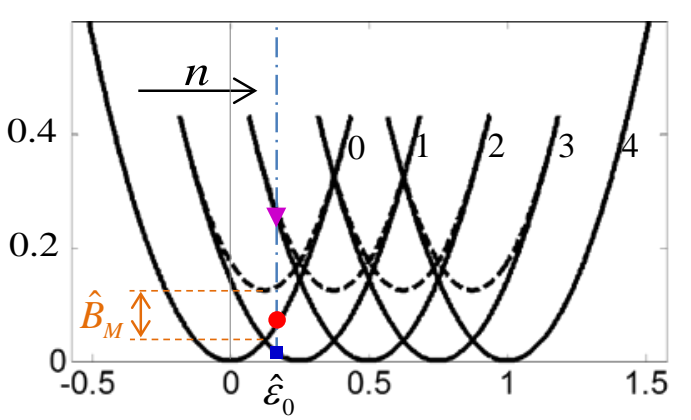

(c)

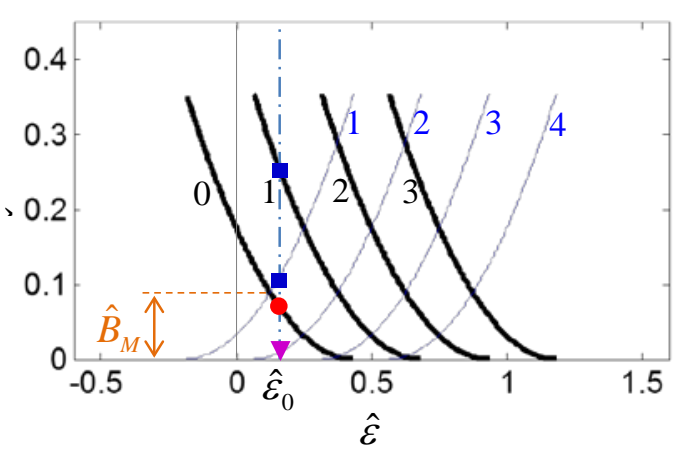

Figure 4: The trilinear chain. (a,b) Equilibrium configurations of a chain composed of four elements $\left(N=4, \lambda=1, \hat{\sigma}_{\min }=-\hat{\sigma}_{\max }, \phi=1.15\right)$. Stable and unstable configurations are indicated by solid and dashed lines, respectively. The filled circle, square and triangle show three different stable configurations, $n=0,1,2$, associated with a prescribed overall strain $\hat{\varepsilon}_{0}$. Note that at this strain, configuration $n=1$ has the lowest energy. (c) Energy barriers for the same chain. Barriers $\Delta F_{n}^{+}$are illustrated with thick black lines (descending lines), while barriers $\Delta F_{n}^{-}$are illustrated with thin blue lines (ascending lines). The barriers associated with the stable configurations, $n=0,1,2$ at overall strain $\hat{\varepsilon}_{0}$ are shown by the circle, square and triangle.

Cross-ref: Fig. 4 


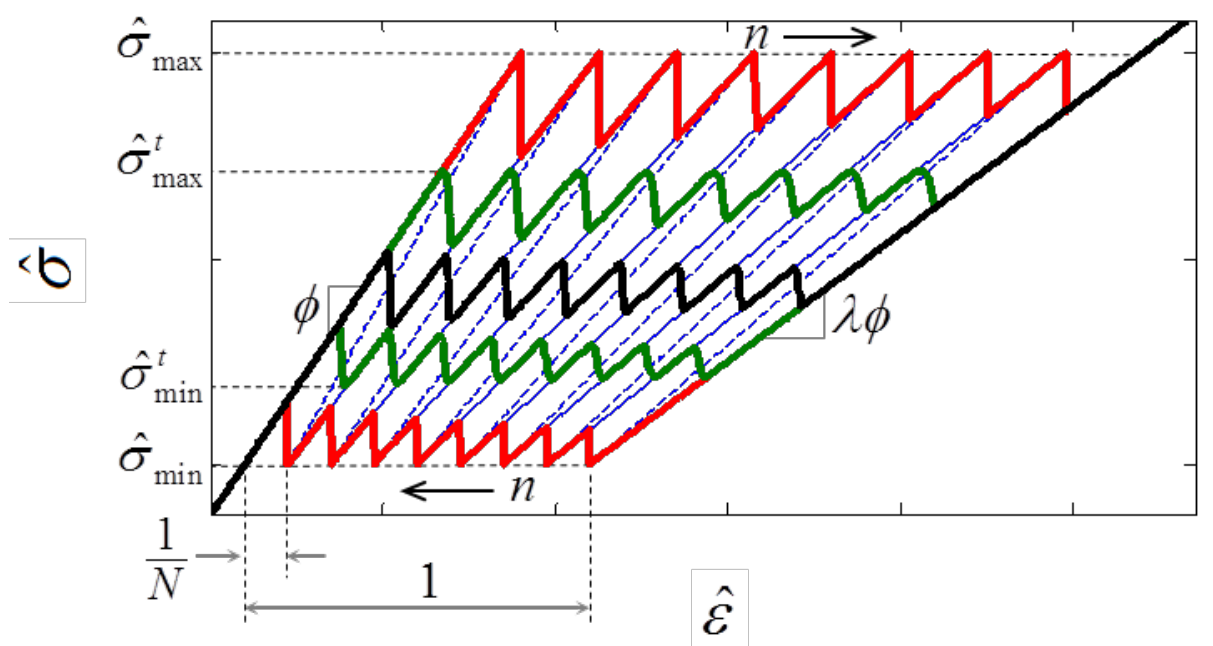

Figure 5: Schematic illustration of typical loading-unloading paths for a trilinear chain with $N=8, \lambda<1, \hat{\sigma}_{\text {max }}=-\hat{\sigma}_{\text {min }}$. The different colors correspond to different temperatures or rates (see text).

Cross-ref: Fig. 5

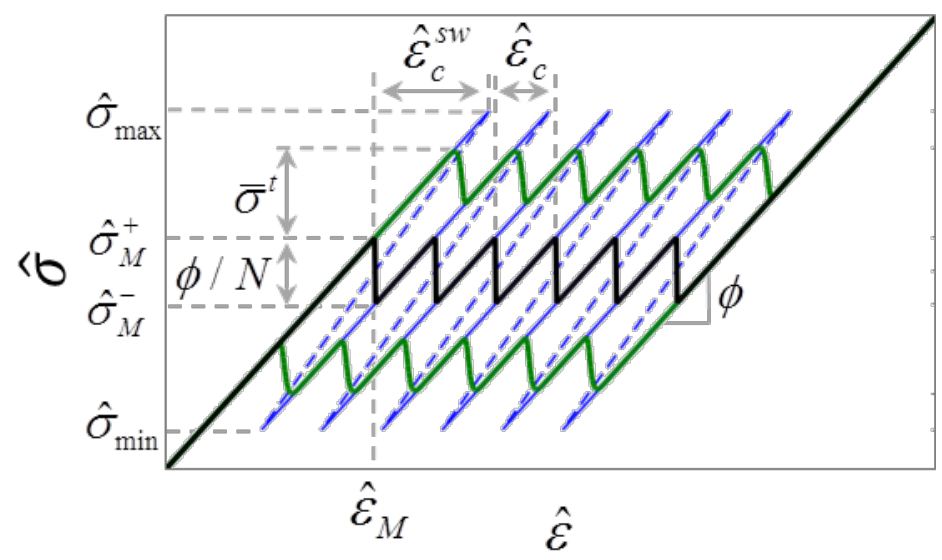

Figure 6: Transition stress on the loading path, $\sigma^{t r}$, is measured with respect to Maxwell's stress, $\sigma_{M}^{+}$. The difference between $t_{c}$ and $t_{c}^{s b}$ is illustrated by $\hat{\varepsilon}_{c}=t_{c} A v / L$ and $\hat{\varepsilon}_{c}^{s w}=t_{c}^{s w} A v / L$.

Cross-ref: Fig. 6 

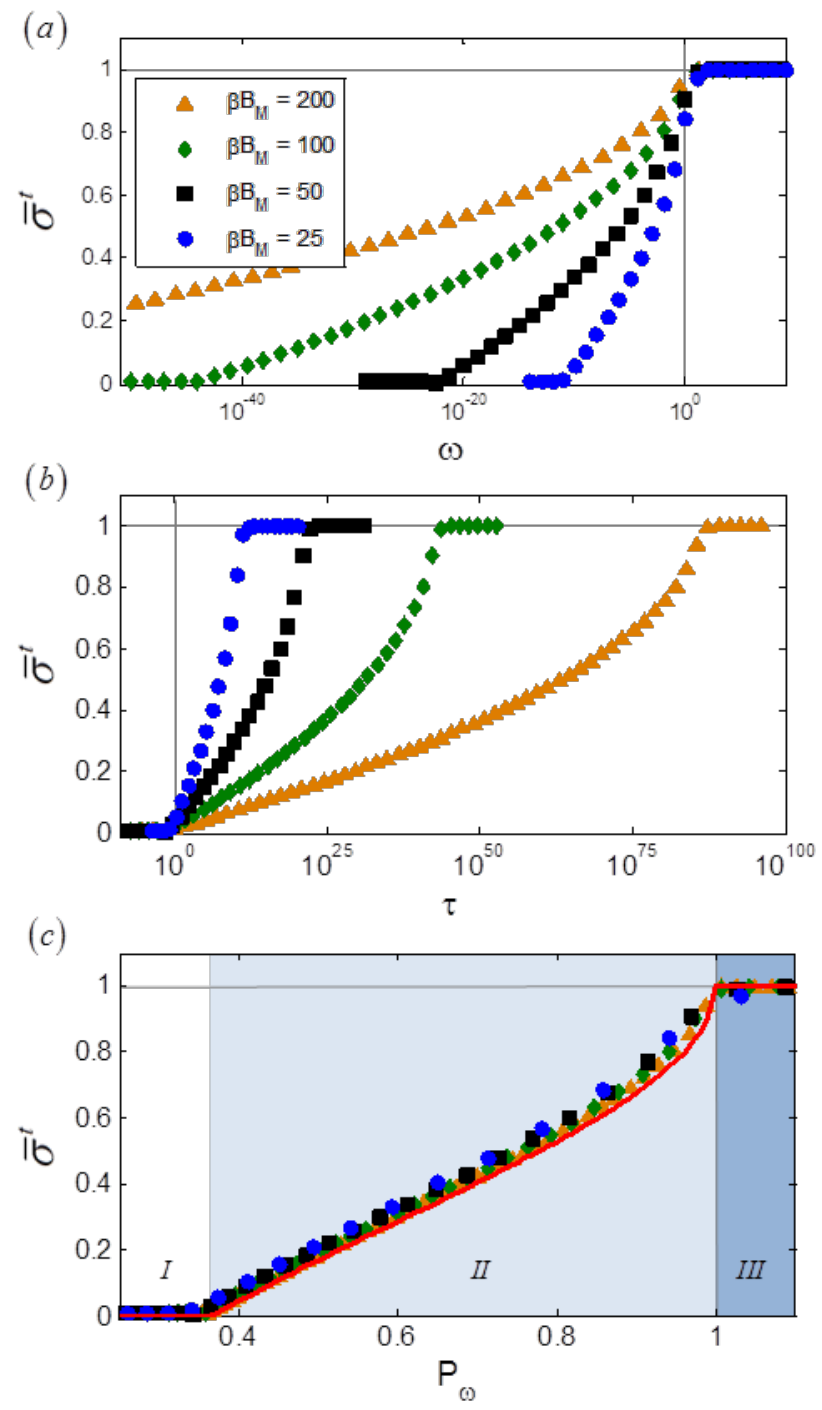

Figure 7: Calculated transition stress for four different temperatures (or relative barriers), $\hat{\beta} \hat{B}_{M}$, and a wide range of rates, $\omega . \hat{\beta} \hat{B}_{M}$ measures the relative height of the typical barrier compared to the thermal energy $k_{B} T$. Here, $N=10, \lambda=1$, $\phi=1.25, \hat{\sigma}_{\min }=-0.5$. (a) As function of $\omega$. (b) As function of $\tau$. (c) With $P_{\omega}$, all sets of data collapse to a single curve with three distinctive regions. Solid line shows relation (5.12).

Cross-ref: Fig. 7 


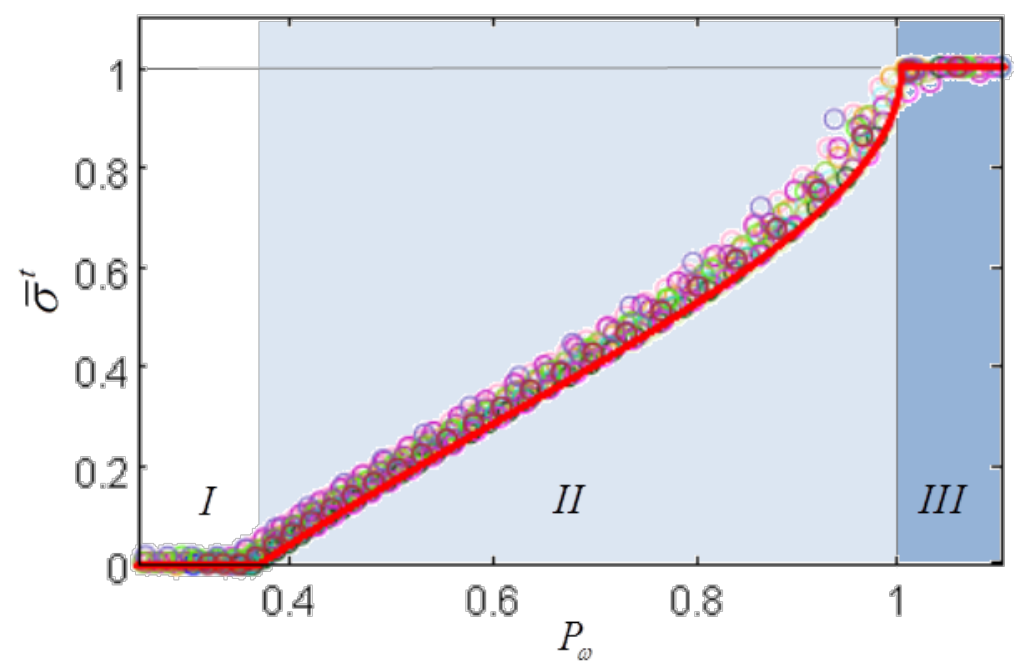

Figure 8: Same as Fig. 7c, but for a wide range of parametric values: $\hat{\beta} B_{M}=25,50,100,200,1000$; $N=5,10,50$; $\lambda=0.1,1,10 ;$ $\phi=0.625,1.25,2.5,5 ; \sigma_{\min }=-0.5,0,0.5$. Solid line shows relation (5.12).

Cross-ref: Fig. 8 

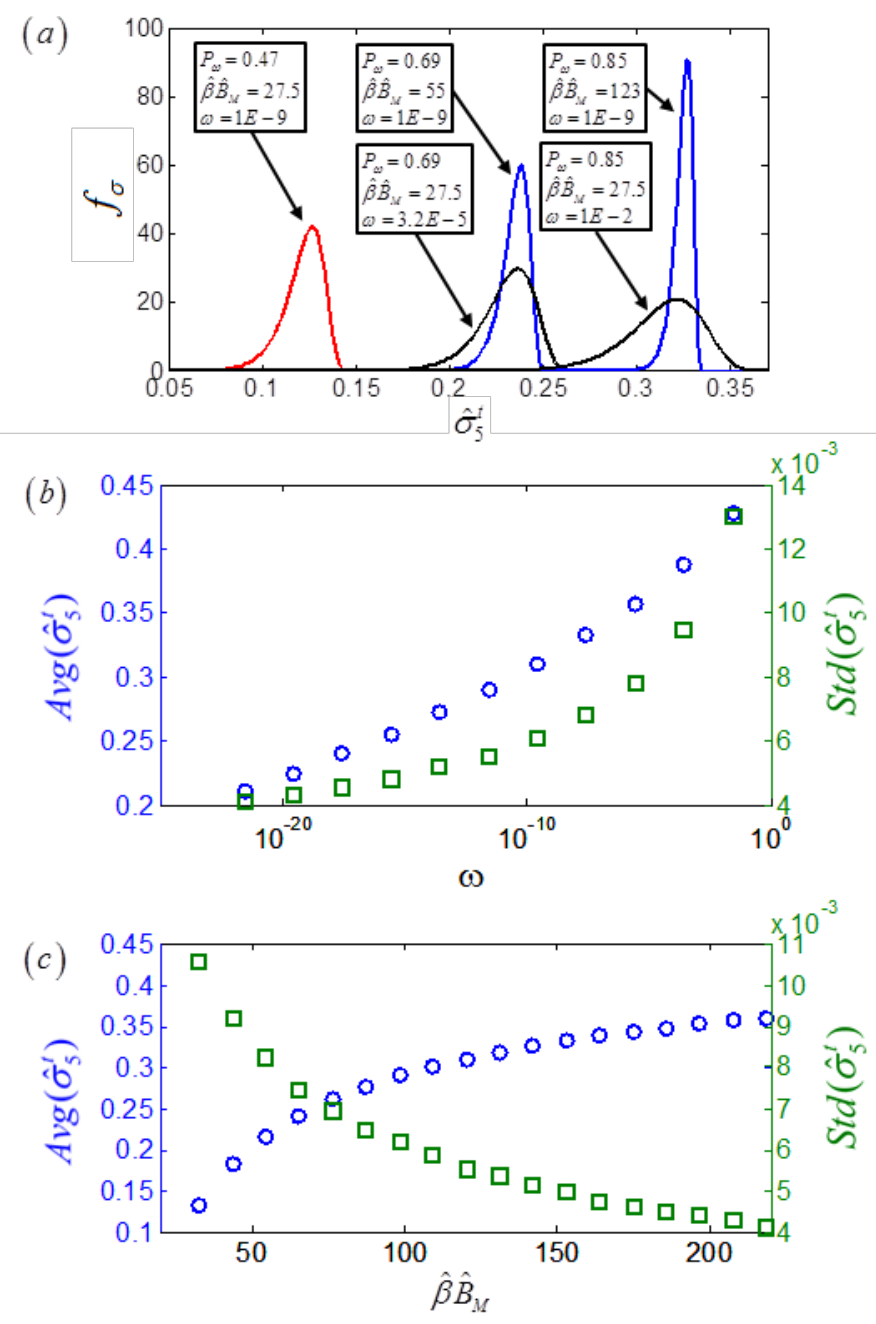

Figure 9: Dispersion of the transition stress for a chain with $N=10, \hat{\sigma}_{\max }=0.5$, $\phi=1.25$. (a) Probability density function, $f_{\sigma}$, for various rates and barriers. (b) Average and standard deviation of the transition stress versus $\omega . \beta B_{M}=123$ (c) Average and standard deviation of the transition stress versus $\beta B_{M} . \omega=10^{-9}$.

Cross-ref: Fig. 9 

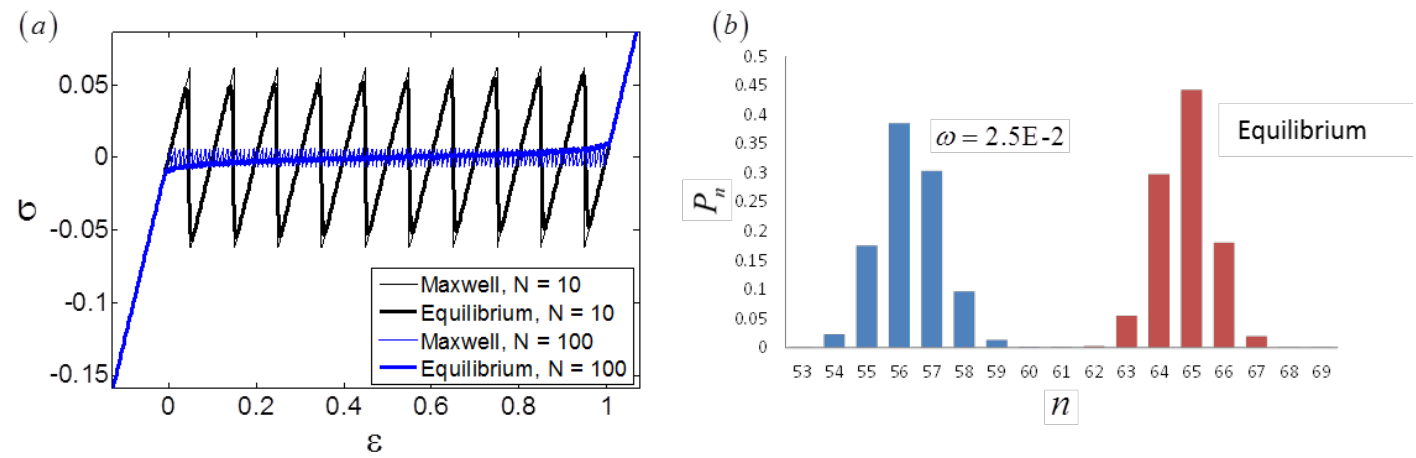

Figure 10: (a) The difference between equilibrium path and Maxwell's path reflected in the stress-strain relations for two identical chains that only differ in the number of elements. (b) Distribution $P_{n}$ at equilibrium and out of equilibrium for the chain with $N=100$.

Cross-ref: Fig. 10
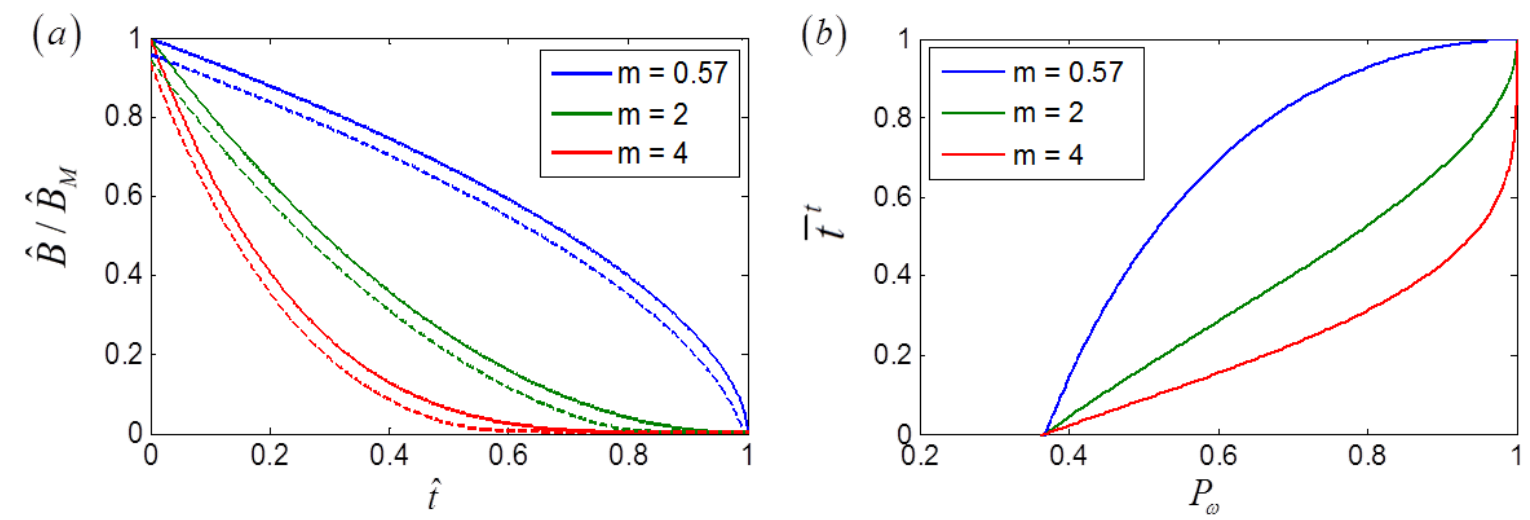

Figure 11: Analytical approximation. (a) Comparison between energy barriers (5.9) and (5.6) illustrated by the dashed and solid lines, respectively. (b) Average transition time (5.7).

Cross-ref: Fig. 11 


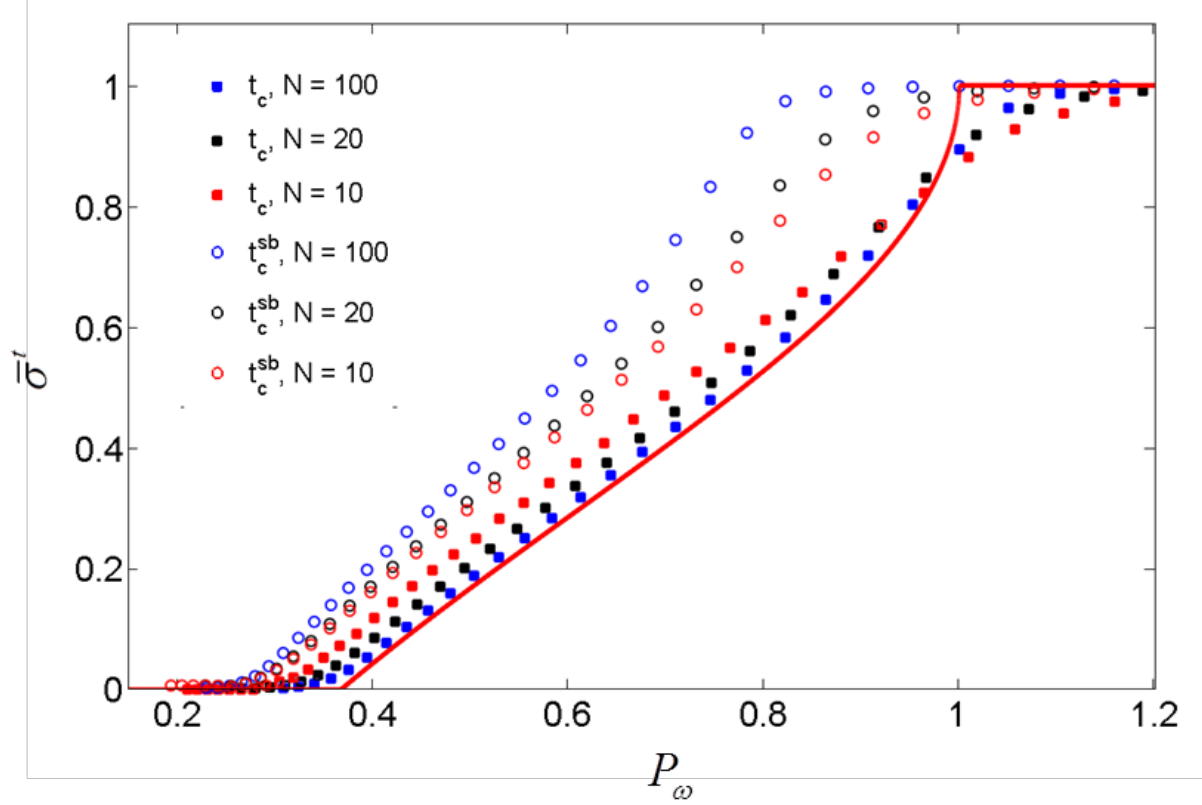

Figure 12: Transition stress versus $P_{\omega}$ using two different timescales, $t_{c}$ (filled squares) and $t_{c}^{s w}$ (circles). $\hat{\beta} \hat{B}_{M} \square 10$. The solid line shows relation (5.12). Results associated with the two time scales coincide for small $N$, and significantly depart for large $N$. Also, for $\hat{\beta} \hat{B}_{M} \square 10$ (not shown here) all results collapse to relation (5.12). Cross-ref: Fig. 12 
(a)

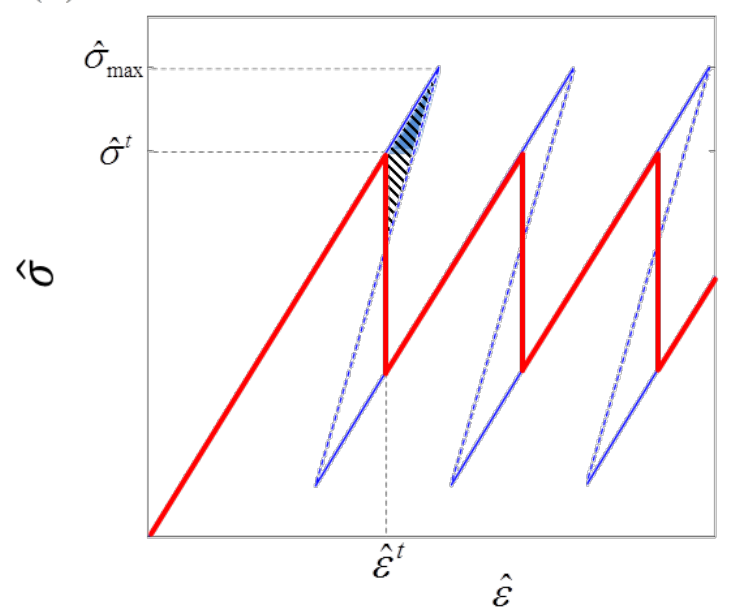

(b)

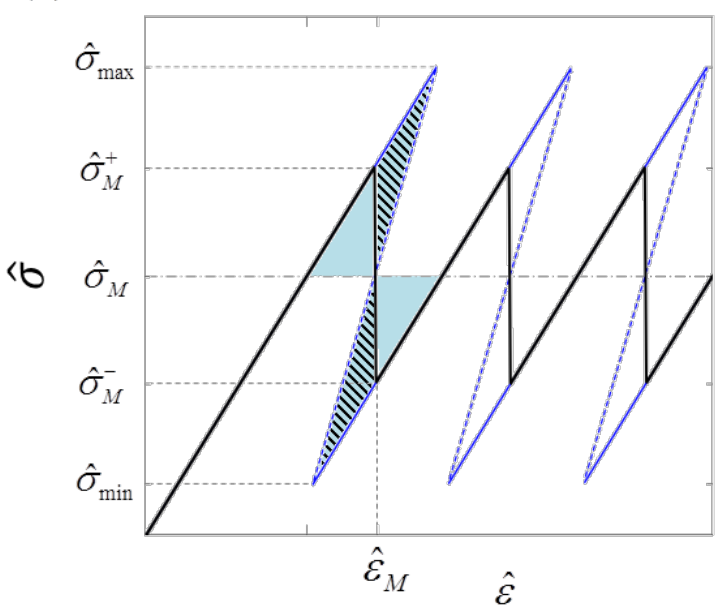

Figure 13: Barriers are different in length-control and force-control conditions. (a) The barrier separating configuration $n=0$ from configuration $n=1$. At the same level of force, the force-control barrier is marked by the shaded area, while the lengthcontrol barrier by the hatched area. (b) For length-control conditions, Maxwell's stress $\hat{\sigma}_{M}^{+}$corresponds to the strain $\hat{\varepsilon}_{M}$ at which the two hatched areas are equal. In force-control conditions, Maxwell's stress $\hat{\sigma}_{M}$ is the stress at which the two shaded areas are equal.

Cross-ref: Fig. 13 